\title{
CONSISTENCY CONDITIONS FOR AFFINE TERM STRUCTURE MODELS
}

\author{
SERGEI LEVENDORSKIǏ \\ Department of Economics, \\ University of Texas at Austin
}

\begin{abstract}
ATSM are widely applied for pricing of bonds and interest rate derivatives but the consistency of ATSM when the short rate, $r$, is unbounded from below remains essentially an open question. First, the standard approach to ATSM uses the Feynman-Kac theorem which is easily applicable only when $r$ is bounded from below. Second, if the tuple of state variables belongs to the region where $r$ is positive, the bond price should decrease in any state variable for which the corresponding coefficient in the formula for $r$ is positive; the bond price should also decrease as the time to maturity increases. In the paper, sufficient conditions for the application of the Feynman-Kac formula, and monotonicity of the bond price are derived, for wide classes of affine term structure models in the pure diffusion case. Necessary conditions for the monotonicity are obtained as well. The results can be generalized for jump-diffusion processes.
\end{abstract}

Key words affine term structure models, Feynman-Kac formula

The author is grateful to Darrel Duffie for the indication that the use of the Feyman-Kac theorem in ATSM is not justified even for many diffusion processes, and illuminating discussions. The author is thankful to the participants of a seminar at the Finance Department of the University of Texas at Austin for useful remarks, and to the referee of the paper for useful suggestions. 


\section{INTRODUCTION}

Consider a financial market under several sources of uncertainty represented by a multi-variate Markov process $X$. The price of an interest rate derivative of the European type, maturing at date $T$, with the terminal pay-off $g(X(T))$, can be expressed as

$$
f(X(t), t)=E_{t}\left[\exp \left(-\int_{t}^{T} r(X(s)) d s\right) g(X(T))\right] .
$$

Starting with one-factor diffusion models Vasicek (1977) and Cox, Ingersoll and Ross (1985), one of the popular approaches has been to model $X$ as the solution to the stochastic differential equation

$$
d X_{j}(t)=b_{j}(X(t), t) d t+\sum_{k=1}^{n} \beta_{j k} \sqrt{S_{j}(X(t), t)} d W_{j},
$$

$j=1, \ldots, n$, where $b_{j}$ and $S_{j}$ are affine functions of $X(t)$, and $d W$ is the increment of the standard $n$-dimensional Brownian motion; $r$ is modelled as an affine function of the state variable:

$$
r(X(s))=\langle d, X(s)\rangle+d_{0}
$$

where $d \in \mathbf{R}^{n}$ is a constant vector, and $d_{0} \in \mathbf{R}$ is a scalar; $\langle\cdot, \cdot\rangle$ denotes the standard inner product in $\mathbf{R}^{n}$. When $r$ is given by (1.3), the Feynman-Kac formula and the Fourier transform can be used to reduce the calculation of $f(X(t), t)$ to the solution of a parabolic equation, and then to solution of a system of ODE (Riccati equations) depending on a parameter, with the subsequent integration w.r.t. this parameter. This idea is due to Heston (1993) who applied it to pricing of bond and currency options. Heston's approach was generalized by Duffie and Kan (1996), who coined the term Affine Term Structure Models (ATSM). For the classification of ATSM under diffusion processes, see Dai and Singleton (2000), and for the extension of ATSM to some jumpdiffusion processes and extensive bibliography on different families of ATSM for both pure jump and jump-diffusion cases, see e.g. Duffie, Pan and Singleton (2000) and Chacko and Das (2002). Notice that the presence of jumps imposes additional restrictions on the parameters of the model. For instance, in one-dimensional case, one must ensure that jumps cannot move $X(t)$ in the region where the volatility coefficient becomes negative. Thus, either the volatility is independent of the state variable, or an appropriate restriction on the direction of jumps

must be imposed. For very general classes of affine Markov models with jumps, under conditions which ensure the non-negativity of $r$, see Duffie, Filipović and Schachermayer (2002). 
The consistency of ATSM in cases when $r$ may be unbounded from below remains essentially an open question. The main stress in the classification paper Dai and Singleton (2000) is on the overdeterminacy of many ATSM models; however, for wide regions in the parameter's space, standard ATSM models may be inconsistent, and the following issues must be addressed. First, the standard approach is based on the Feynman-Kac formula but the general Feynman-Kac theorem is formulated for bounded (and sufficiently regular, say, continuous) $r$ and sufficiently regular $g$; an extension to the case of $r$ which are bounded from below is straightforward. Thus, the first step of the solution of an ATSM, namely, the reduction to the backward parabolic problem

$$
\begin{aligned}
\left(\partial_{t}+L-r\right) f(x, t) & =0, \quad t<T ; \\
f(x, T) & =g(x),
\end{aligned}
$$

where $L$ is the infinitesimal generator of $X$, cannot be easily deduced from a general Feyman-Kac theorem unless the affine $r$ depends on $X_{j}$ of the CIR-type only ${ }^{1}$. If some of $X_{j}$ may assume arbitrary (real) values, and the corresponding coefficients $d_{j}$ in (1.3) are non-zero, then $r$ is unbounded from below, and to the best of our knowledge, no universal statement exists which guarantees that the solution to the problem (1.4)-(1.5), call it $f_{0}(g, r ; x, t)$, coincides with $f(g, r ; x, t)$ given by the stochastic expression (1.1). Second, it is natural to assume that in the fully consistent model, the solution to the bond pricing problem must be a decreasing function of any state variable for which the corresponding coefficients in the formula for $r$ are positive; the solution must also decrease as the time to maturity increases, if the tuple of state variables belongs to the region where $r$ is positive.

In the classification of Dai and Singleton (2000), a model is said to belong to family $A_{m}(n)$ if the number of the factors of the CIR-type is $m$. The two opposite cases, $m=n$ and $m=0$, are especially simple. If $m=n$, then the justification of the use of the Feynman-Kac theorem is a special case of the general result, and if in addition, $d_{0} \geq 0$, then the monotonicity of the bond price is evident from (1.1). If $m=0$, then $X$ is the Ornstein-Uhlenbeck process on $\mathbf{R}^{n}$, for which an explicit formula for the characteristic function $E^{x}\left[e^{i\langle\xi, X(t)\rangle}\right]$ is available (see equation $(17.4)$ in Sato $\left.(1999)^{2}\right)$. The formula holds even for a wider class of processes of Ornstein-Uhlenbeck type, which are driven by Lévy processes, and it is equivalent to the statement that the stochastic expression (1.1)

\footnotetext{
${ }^{1}$ We say that a factor $X_{j}$ in an ATSM is of the CIR-type iff it assumes values in $\mathbf{R}_{+}$

${ }^{2}$ The author is grateful to Darrell Duffie for the reference
} 
with $g \equiv 1$ is equal to the solution to the Riccati equations for the bond price, that is, $f_{0}(1, r ; x, t)=f(1, r ; x, t)$. However, there is no reason to expect that the bond price is monotone in this case for all parameters' values. For $1 \leq m \leq n-1$, both the reduction to the Riccati equations and the monotonicity conditions have not been studied.

Let $\tau=T-t$ be the time to maturity, and

$$
P(x, \tau)=\exp \left[\sum_{j=1}^{n} B_{j}(\tau) x_{j}+C(\tau)\right]
$$

be the price of the bond, which is obtained in an ATSM model by the formal reduction to the Riccati equations. First, we consider the Vasicek model and its generalization, namely, family $A_{0}(n)$, then a simple two-factor $A_{1}(2)$ model, next more general $A_{1}(n)$ model, and finally the family $A_{2}(3)$ (other families $A_{m}(n)$ can be studied similarly), and derive, in terms of parameters of the model,

(I) simple necessary conditions for the decay of $P(x, \tau)$ :

$$
P_{\tau}^{\prime}(x, \tau)<0, \quad \forall x \quad \text { s.t. } r(x) \geq 0, \quad \text { and } \forall \tau>0 ;
$$

in some cases, we also show that these conditions imply the boundedness of the bond price: in the region $\left\{x \mid x_{j}>0\right.$ : $\left.d_{j}>0\right\}$,

$$
P(x, \tau)<C, \quad \forall \tau>0 ;
$$

(II) sufficient conditions for the decay of $B_{j}(\tau)$; we do not know how wide is the gap between these conditions and the (unknown to us) necessary and sufficient conditions;

(III) sufficient conditions under which the reduction to the system of the Riccati equations can be justified. For $A_{1}(2)$ family, and in many other cases, these condition are weaker than the necessary conditions in (I).

Remark 1.1. a) As it was mentioned above, for $A_{0}(n)$-model, the reduction to the system of Riccati equations is known, and it is valid without additional conditions on parameters of the model.

b) Necessary and sufficient conditions for (1.7) in a neighborhood of $\tau=+\infty$, and in a vicinity of 0 , are easier to derive, and under these conditions, a "numerical proof" of the monotonicity of the bond price on a large finite interval can be used to show that for given parameters' values, the model is consistent.

c) As our study shows, for family $A_{1}(n)$, the monotonicity of $P(x, \tau)$ w.r.t. $\tau$ is the main consistency problem for ATSM (and the only consistency problem for family $\left.A_{0}(n)\right)$. On the other hand, should one use the model for a fixed (and sufficiently small) time to maturity then 
the model can be consistent on this time interval; and it is possible to derive sufficient conditions for (1.7) to be valid on a sufficiently narrow interval $\left(0, \tau_{0}\right)$, where $\tau_{0}>0$ depends on parameters of the model.

d) When it is necessary to consider more general contingent claims, a sufficient condition for (III), in terms of the rate of growth of the pay-off at infinity, can be derived relatively easily, and the same is true of a necessary condition for the natural analog of (1.7) and sufficient condition for (1.8). The sufficient conditions for the monotonicity will be more difficult to derive.

e) It is plausible that in some empirical studies, the fitted ATSM is inconsistent in the sense that the monotonicity condition fails. Hence, if the model is fitted for some time to maturity, and used later for a larger time, then it may produce non-monotonic bond prices.

f) Similar consistency problems exist for interest rate derivative products, and an unnatural behavior of the price of a derivative product can be easily overlooked if one fits the parameters of the model by using the data on bond prices, and then uses the calibrated model to calculate prices of interest rate derivatives. It might be possible to construct an arbitrage strategy against a counterparty who uses an inconsistent model.

One may argue that the consistency analysis should be conducted when not only parameters of the model are fixed but values of (unobservable) factors as well: if for a chosen set of the parameters and factors the bond price is a decaying function of $\tau$, then the model is reasonable. However, it is not clear how to obtain a general result in this set-up, and moreover, it does not seem to be right to put the factors on the equal footing with the parameters of the model, especially in cases when the factors can be interpreted as the short rate, its volatility and/or central tendency.

We assume that the model should be consistent for all positive values of factors, the interest rate depends on (we consider the case when in (1.3), the coefficients $d_{j}, j=1, \ldots, n$, are non-negative), which makes the model less flexible than it is assumed in Dai and Singleton (2000). For the factors of the CIR-type, our restriction on $d_{j}$ 's is natural, and it is without loss of generality as far as the factors assuming values in $\mathbf{R}$ are concerned: if one of these $d_{j}$ is negative, one can make it positive by using $-X_{j}$ instead of $X_{j}$. On the other hand, the model specifications are underdetermined in the sense of Dai and Singleton (2000) because this allows us to formulate necessary conditions and sufficient ones in more symmetric and natural forms.

The rest of the paper is organized as follows. In Section 2, we formulate and prove the results for the Vasicek model and its generalization, 
$A_{0}(n)$ - model. Although the justification of the use of the Feyman-Kac theorem can be made by appealing to formula (17.4) in Sato (1999), we give an independent proof of the reduction. The main idea of the proof is the conjugation with an appropriate exponent, which allows us to reduce to the case of an interest rate bounded from below. The same idea is used in the proofs for $A_{m}(n)$-models, $m \geq 1$, however the realization becomes much more involved, if we want to avoid too stringent conditions.

In Section [3, we formulate the main results for the case $m \geq 1$, and prove theorems about properties of the formal solution (1.6) of the ATSM model. In Section 4, we describe the general scheme of justification of the reduction to the backward parabolic equation; in other words, the scheme of the proof of the Feynman-Kac formula for an affine $r$ and the class of processes used in $A_{m}(n)$ models, $m \geq 1$. The main ingredient of the proof is the representation theorem for analytic semigroups. The proof of the reduction for $A_{1}(n)$ model and $A_{2}(3)$ model is given in Section 5 (more general $A_{m}(n)$-models can be studied similarly). The most technical part of the proof, namely, the proof of the existence and uniqueness theorem for degenerate elliptic operators with parameter is delegated to the appendix. The proof of the latter theorem uses a general approach to problems of this sort described in detail in the review paper Levendorskii and Paneyakh (1990) and monograph Levendorskii (1993). This approach is applicable not to differential operators only but to integro-differential operators (another name: pseudo-differential operators) as well, which allows one to justify the use of the Feynman-Kac formula for jump-diffusion processes. This more general case will be treated in a separate publication.

\section{FAMiLY $A_{0}(n)$}

2.1. Monotonicity conditions. The interest rate is given by (1.3) with non-negative $d_{j}, j \geq 1$, and positive $d_{n}$, and the dynamics of $X(t)$ is given by $\mathrm{SDE}$

$$
d X(t)=(\theta-\kappa X(t)) d t+\Sigma d W(t)
$$

where $W(t)$ is the standard Brownian motion in $\mathbf{R}^{n}, \kappa=\left[\kappa_{j l}\right]$ is a low-diagonal matrix with positive diagonal elements, $\Sigma$ is a positivedefinite matrix, and $\theta$ is a constant vector. The bond price is given by (1.6) with

$$
B(\tau)=-\left(1-\exp \left(-\tau \kappa^{T}\right)\right) \gamma
$$


where $\gamma=\left(\kappa^{T}\right)^{-1} d$, and

$$
C(\tau)=\int_{0}^{\tau}\left(-d_{0}+\theta^{T} B(s)+\frac{1}{2} \operatorname{Tr}\left(\Sigma^{T}\left[B_{j}(s) B_{l}(s)\right]_{j, l=1}^{n} \Sigma\right)\right) d s,
$$

and necessary and sufficient conditions for the decay of the bond price are (relatively) easy to establish. These conditions are especially simple in the case $n=1$ (the Vasicek model).

Theorem 2.1. Let $n=1$, and let the dynamics of the short rate be given by

$$
d r(t)=(\theta-\kappa r(t)) d t+\sigma d W(t),
$$

where $\theta, \kappa$ and $\sigma$ are positive constants.

Then a) if for some $r>0$, the bond price $P(r, \tau)$ is a non-increasing function of $\tau$, then

$$
\frac{\sigma^{2}}{2} \leq \kappa \theta
$$

b) if (2.4) holds, then for any $r>0$, the bond price $P(r, \tau)$ is a decreasing function in $\tau$.

Proof. Clearly, $B(\tau)=-\kappa^{-1}\left(1-e^{-\kappa \tau}\right)$ is decreasing on $[0,+\infty)$ from 0 to $-\kappa^{-1}$, therefore if

$$
C^{\prime}(\tau)=\theta B(\tau)+\frac{\sigma^{2}}{2} B(\tau)^{2}
$$

is non-positive on $(0,+\infty)$, then (2.4) holds, and if (2.4) holds, then $C^{\prime}(\tau)$ is negative on $(0,+\infty)$.

Condition (2.4) means that for a given central tendency and coefficient of mean reversion, the volatility may not be too large, and the interpretation is clear: if volatility is large, then a trajectory of the process spends significant amount of time in a region of the state space where the short rate is negative, which leads to the artificial increase of the bond price.

In the case $n \geq 2$, it is convenient to study the monotonicity of $B_{j}$ step by step, by using the low-diagonal structure of $\kappa$, instead of appealing to the explicit general formula (2.2). Due to positivity of the diagonal elements of matrix $\kappa$ and the assumption $d_{n}>0$, the $B_{n}$ is decreasing from 0 to $-d_{n} / \kappa_{n n}$. Hence, if

$$
B_{n-1}(\tau)=\int_{0}^{\tau} e^{-\kappa_{n-1, n-1}(\tau-s)}\left(-\kappa_{n, n-1} B_{n}(s)-d_{n-1}\right) d s
$$

is non-increasing on $(0,+\infty)$, then it must be that

$$
d_{n-1} \kappa_{n n}-d_{n} \kappa_{n, n-1} \geq 0
$$


and if (2.5) holds, then $B_{n-1}$ decreases. For $B_{j}, j \leq n-2$, the necessary conditions for the monotonicity are not that simple but the induction and the same consideration as above show that if the off-diagonal entries of the matrix $\kappa$ are non-positive then all $B_{j}$ 's are decreasing functions. From (2.3), it is evident that then the bond price is a decreasing function in $\tau$ if and only if the function

$$
\mathbf{R}_{+} \ni s \mapsto F(s):=-d_{0}+\langle\theta, B(s)\rangle+\frac{1}{2} \operatorname{Tr}\left(\Sigma^{T}\left[B_{j}(s) B_{l}(s)\right]_{j, l=1}^{n} \Sigma\right) \in \mathbf{R}
$$

is non-positive. Clearly, necessary and sufficient conditions for nonpositivity of $F$ in terms of the parameters of the model cannot be simple, however, relatively simple necessary conditions and sufficient ones (the latter more stringent than the former) are easy to formulate. From (2.2), we find that $B(+\infty)=-\gamma:=-\left(\kappa^{T}\right)^{-1} d$, and therefore for any $j=1, \ldots, n$, and $s>0$, we have $B_{j}(s) \in\left(-\gamma_{j}, 0\right)$. Introduce the quadratic polynomial

$$
Q(y)=-d_{0}+\langle\theta, y\rangle+\frac{1}{2} \operatorname{Tr}\left(\Sigma^{T}\left[y_{j} y_{l}\right]_{j, l=1}^{n} \Sigma\right) .
$$

If $Q(-\gamma)>0$, then for sufficiently large $s, F(s)>0$, and therefore $C$ grows in a neighborhood of $+\infty$; this gives a necessary condition

$$
Q(-\gamma) \leq 0
$$

Since the quadratic form in the definition of $Q(y)$ is positive-definite, $Q$ is non-positive on $U^{\gamma}:=\left[-\gamma_{1}, 0\right] \times \cdots \times\left[-\gamma_{n}, 0\right]$ (which implies that the integrand in (2.3) is negative a.e., and $C$ decreases) if and only if it is non-positive at each vertex of $U^{\gamma}$, that is,

$$
Q(y) \leq 0 \quad \forall y \in\left\{-\gamma_{1}, 0\right\} \times \cdots \times\left\{-\gamma_{n}, 0\right\} .
$$

We have obtained

Theorem 2.2. a) Let $n=2$, and let $B_{1}$ be non-increasing.

Then (2.5) holds.

b) Let $n \geq 2$, and let all $B_{j}$ be non-increasing. Then (2.6) holds.

c) Let $n \geq 2$, let the off-diagonal entries of $\kappa$ be non-positive, and let (2.7) hold.

Then the bond price is a decreasing function of $(x, \tau)$ in the region $x>0, \tau>0$.

2.2. Justification of the Feyman-Kac formula. We assume that $\Sigma$ is non-degenerate.

Theorem 2.3. Let $g$ be a continuous function, which does not grow too rapidly at the infinity

$$
\ln (1+|g(x)|)=o\left(\|x\|^{2}\right), \quad \text { as } x \rightarrow+\infty .
$$


Then the expressions (1.1), (1.2) and (1.3) are the unique solution to the problem (1.4)-(1.5) in the class of continuous functions, which admit the bound (2.8) uniformly in $t \in[0, T]$.

As it will be seen from the proof, condition on $g$ can be relaxed, and the solution is unique in a wider class of functions.

Proof. By making an affine transformation of the factors, we may assume that there exist $c_{0}>0$ such that

$$
\langle\kappa x, x\rangle \geq c_{0}\|x\|^{2} \text {. }
$$

Denote by $f_{0}(g, r, ; x, t)$ a solution to the problem (1.4)-(1.5) .

Lemma 2.4. a) Let $r$ and $g$ be continuous, let $g$ satisfy (2.8) and $r$ satisfy

$$
r(x)=o\left(\|x\|^{2}\right), \quad \text { as } x \rightarrow \infty .
$$

Then a solution to the problem (1.4)-(1.5) in the class of continuous functions $f(x, t)$, which admit the bound (2.8) uniformly in $t \in[0, T]$, exists and it is unique.

b) Let $r_{1}, r_{2}, \ldots, r_{N}, \ldots$, be a sequence of continuous functions which satisfies (2.10) uniformly in $N$, and converges pointwise to a function $r$. Then

$$
f_{0}\left(g, r_{N} ; x, t\right) \rightarrow f_{0}(g, r ; x, t) \quad \text { as } \quad N \rightarrow+\infty
$$

pointwise.

Proof. The key element of our approach is the conjugation with an appropriate exponential function; in the case of $A_{0}(n)$, this function is especially simple. Take a small $\epsilon>0$, and set

$$
\begin{aligned}
f_{\epsilon}(g, r ; x, t) & =\exp \left(-\epsilon\|x\|^{2}\right) f_{0}(g, r ; x, t), \\
g_{\epsilon}(x) & =\exp \left(-\epsilon\|x\|^{2}\right) g(x) .
\end{aligned}
$$

Similarly, define $f_{\epsilon}\left(g, r_{N} ; x, t\right)$. Due to (2.8) $, f_{\epsilon}(g, r ; x, t), f_{\epsilon}\left(g, r_{N} ; x, t\right)$ and $g_{\epsilon}(x)$ vanish as $x \rightarrow \infty$, uniformly in $N$ and $t \in[0, T]$.

a) Insert $f_{0}(g, r ; x, t)=\exp \left(\epsilon\|x\|^{2}\right) f_{\epsilon}(g, r ; x, t)$ into (1.4)-(1.5), and multiply by $\exp \left(-\epsilon\|x\|^{2}\right)$. The result is a problem of the same form

$$
\begin{aligned}
\left(\partial_{t}+L_{\epsilon}-r_{\epsilon}\right) f_{\epsilon}(g, r, x, t) & =0, \quad t<T ; \\
f_{\epsilon}(g, r, x, T) & =g_{\epsilon}(x),
\end{aligned}
$$

where $r_{\epsilon}$ is a function, and $L_{\epsilon}$ is a differential operator without the first order term, which are obtained from

$$
\exp \left(-\epsilon\|x\|^{2}\right)(L-r) \exp \left(\epsilon\|x\|^{2}\right)=L_{\epsilon}-r_{\epsilon} .
$$

It is easily seen that the coefficients of $L_{\epsilon}$ at derivatives of order 2 are the same as the ones of $L$, and the other coefficients of $L_{\epsilon}$ tend to the 
corresponding coefficients of $L$ as $\epsilon \rightarrow 0$. Hence, if $\epsilon>0$ is sufficiently small, then $L_{\epsilon}$ is the infinitesial generator of an Ornstein-Uhlenbeck process, call it $X^{\epsilon}$. Fix such an $\epsilon$. Further, $r_{\epsilon}=r+\tilde{r}_{\epsilon}$, where

$$
\tilde{r}_{\epsilon}(x)=2 \epsilon\langle\kappa x, x\rangle+\epsilon\left(\operatorname{Tr}\left(\Sigma^{T} \Sigma\right)-2\langle\theta, x\rangle\right),
$$

and in view of (2.10) and (2.9), there exist positive $c, C$ such that

$$
c\|x\|^{2}-C \leq r_{\epsilon}(x) \leq c\|x\|^{2}+C, \quad \forall x \in \mathbf{R}^{n} .
$$

Since $r_{\epsilon}$ is bounded from below, the solution to problem (2.12)-(2.13) exists in the class of continuous functions decaying as $x \rightarrow \infty$, and it is unique (in fact, the solution is unique in the class of functions which grow not faster than an exponential function). Since $f_{0}(g, r ; x, t)=$ $\exp \left(\epsilon\|x\|^{2}\right) f_{\epsilon}(g, r ; x, t)$, part a) has been proved.

b) The argument above is applicable with $r_{N}$ instead of $r$, and $r_{N, \epsilon}=r_{N}+\tilde{r}_{\epsilon}$ admits bound (2.14) uniformly in $N$. It follows that $f_{\epsilon}\left(g, r_{N}, x, t\right)$ is given by the Feynman-Kac formula

$$
f_{\epsilon}\left(g, r_{N}, x, t\right)=E_{t}\left[\exp \left(-\int_{t}^{T} r_{N, \epsilon}\left(X^{\epsilon}(s)\right) d s\right) g_{\epsilon}\left(X^{\epsilon}(T)\right)\right] .
$$

Since $r_{N, \epsilon}(x) \rightarrow r_{\epsilon}(x)$, as $N \rightarrow+\infty$, point-wise, we use the Dominant Convergence Theorem, pass to the limit in (2.15), and obtain that $f_{\epsilon}\left(g, r_{N}, x, t\right) \rightarrow f_{\epsilon}(g, r, x, t)$, point-wise. It follows that $f_{0}\left(g, r_{N}, x, t\right) \rightarrow$ $f_{0}(g, r, x, t)$, point-wise.

Now we give the proof of Theorem [2.3. Denote $f(g, r ; x, t)$ as the stochastic expression (1.1). Fix $N \in \mathbf{R}$, and for the affine $r$, set $r_{N}(x)=\max \{r(x),-N\}$. Then $r_{N}$ is bounded from below, hence both $f\left(g, r_{N} ; x, t\right)$ and $f_{0}\left(g, r_{N} ; x, t\right)$ exist, and $f\left(g, r_{N} ; x, t\right)=f_{0}\left(g, r_{N} ; x, t\right)$ for all $x$ and $t<T$. Since $g, r_{N}$ and $r$ satisfy the conditions of Lemma 2.4. (2.11) holds. By the Monotone Convergence Theorem, $f\left(g, r_{N} ; x, t\right) \rightarrow f(g, r ; x, t)$ as $N \rightarrow+\infty$, point-wise, and we conclude that $f(g, r ; x, t)=f_{0}(g, r ; x, t)$ for all $x$ and $t \leq T$.

\section{FAMilies $A_{m}(n), 1 \leq m \leq n-1$.}

3.1. Family $A_{1}(2)$. The state space is $\mathbf{R}_{+} \times \mathbf{R}$, the $r$ is given by (1.3) with $d_{1} \geq 0, d_{2}>0$, and the infinitesimal generator of the process is of the form

$$
L=\left(\theta_{1}-\kappa_{11} x_{1}\right) \partial_{1}+\left(\theta_{2}-\kappa_{21} x_{1}-\kappa_{22} x_{2}\right) \partial_{2}+\frac{1}{2} x_{1} \partial_{1}^{2}+\frac{\alpha+\beta x_{1}}{2} \partial_{2}^{2},
$$

where $\kappa_{11}, \kappa_{22}, \theta_{1}, \alpha, \beta$ are positive. Without loss of generality, $\theta_{2}=0$.

Assume that in the case of the bond, the use of the Feynman-Kac theorem has been justified. Then $P(x, \tau)=f_{0}(1, r ; x, t)$, the solution 
to (1.4) -(1.5) with $g(x) \equiv 1$, can be found in the form (1.6). By substituting (1.6) into (1.4)-(1.5), we obtain the system of Riccati equations on $(0, T)$ :

$$
\begin{aligned}
B_{1}^{\prime} & =-\kappa_{11} B_{1}+\frac{1}{2} B_{1}^{2}-\kappa_{21} B_{2}+\frac{\beta}{2} B_{2}^{2}-d_{1}, \\
B_{2}^{\prime} & =-\kappa_{22} B_{2}-d_{2}, \\
C^{\prime} & =-d_{0}+\theta_{1} B_{1}+\frac{\alpha}{2} B_{2}^{2},
\end{aligned}
$$

subject to the boundary conditions

$$
B_{1}(0)=0 ; \quad B_{2}(0)=0 ; \quad C(0)=0 .
$$

We solve (3.3) subject to $B_{2}(0)=0$ :

$$
B_{2}(\tau)=-\gamma\left(1-e^{-\kappa_{22} \tau}\right)
$$

where $\gamma:=\kappa_{22}^{-1} d_{2}>0$. $B_{2}$ decreases from 0 to $-\gamma$, since $\kappa_{22}>0$ :

$$
\lim _{\tau \rightarrow+\infty} B_{2}(\tau)=-\gamma<0
$$

If $B_{1}\left(\tau_{0}\right)>0$ for some $\tau_{0}>0$, then not only $P(x, \tau)$ fails to be a decaying function of $\tau$; for this $\tau_{0}, P(x, \tau)$ is an increasing function in $x_{1}$. The following theorem provides necessary conditions which exclude such a strange behavior of the bond price (the higher the spot short rate, the higher the price of the bond), and sufficient conditions for the negativity of $B_{1}(\tau)$ for $\tau>0$.

Theorem 3.1. a) If $B_{1}$ is non-increasing on $[0,+\infty)$, then the following two conditions hold:

$$
\begin{aligned}
& d_{1}>0 \quad \text { or } \quad \kappa_{21}<0 \\
& d_{1}-\kappa_{21} \gamma-\frac{\beta}{2} \gamma^{2} \geq 0
\end{aligned}
$$

b) If (3.8)-(3.9) hold, then

$$
B_{1}(\tau)<0, \quad \forall \tau>0 .
$$

Proof. a) Suppose that $d_{1}=\kappa_{21}=0$ but $B_{1}(\tau)$ is non-positive in a right neighborhood of 0 . From (3.6), the RHS in (3.2) is positive in this neighborhood, hence $B_{1}$ is increasing in this neighborhood from 0 . Hence, $B_{1}$ is positive there; contradiction. Thus, (3.8) holds.

Denote by $\hat{d}$ the LHS of (3.9). In view of (3.7), the RHS of (3.2) admits the representation

$$
-\kappa_{11} B_{1}(\tau)+\frac{1}{2} B_{1}(\tau)^{2}-\hat{d}+o(1), \quad \text { as } \tau \rightarrow+\infty .
$$


Hence, if $\hat{d}$ is negative, and $B_{1}$ stays negative, then eventually, the RHS of (3.2) will exceed a positive constant, and $B_{1}$ will grow as a linear function of $\tau$, contradiction.

b) From (3.8) and (3.6), we see that $B_{1}$ is negative in a right neighborhood of 0 . Consider the quadratic polynomial $Q(y)=-d_{1}-\kappa_{21} y+\frac{\beta}{2} y^{2}$. Under condition (3.8), it is negative in a small left neighborhood of 0 , therefore if (3.9) holds, it is negative on $(-\gamma, 0)$. Hence,

$$
-d_{1}-\kappa_{21} B_{2}(\tau)+\frac{\beta}{2} B_{2}(\tau)^{2}<0, \quad \forall \tau>0 .
$$

We conclude that if $B_{1}(\tau)$ tries to approach zero from below as $\tau$ increases, then the RHS in (3.2) becomes negative, and pushes the trajectory $\tau \mapsto B_{1}(\tau)$ down. Hence, $B_{1}(\tau)$ remains negative.

The sufficient conditions for the monotonicity are stronger than (3.8)(3.9); in fact, additional necessary conditions can be derived. We are satisfied with (3.8)-(3.9) for the time being because these conditions are more restrictive than the sufficient conditions for the use of the Feynman-Kac formula in Theorem 3.3 below. The following theorem provides sufficient conditions for the decay of $B_{1}$ and $C$.

Theorem 3.2. a) Let (3.8) and (3.9) hold, and let

$$
\beta \gamma+\kappa_{21} \leq 0
$$

Then $B_{1}$ decreases on $[0,+\infty)$.

b) Let (3.8), (3.9) and (3.11) hold, and let

$$
d_{0} \geq \frac{\alpha}{2} \gamma^{2}
$$

Then $C$ decreases on $[0,+\infty)$, and the bond price is a decreasing function of $(x, \tau)$ in the region $x_{1}, x_{2}>0, \tau>0$.

Proof. a) Set $Y=B_{1}^{\prime}$, and solve (3.2) w.r.t. $B_{1}$ taking into account that the latter is negative:

$$
B_{1}=\kappa_{11}-\sqrt{\kappa_{11}^{2}+2 \kappa_{12} B_{2}-\beta B_{2}^{2}+2 d_{1}+2 Y} .
$$

By differentiating (3.2), we find

$$
\begin{aligned}
Y^{\prime} & =-\kappa_{11} Y+Y B_{1}-\kappa_{21} B_{2}^{\prime}+\beta B_{2} B_{2}^{\prime} \\
& =-Y \sqrt{\kappa_{11}^{2}+2 \kappa_{12} B_{2}-\beta B_{2}^{2}+2 d_{1}+2 Y}+B_{2}^{\prime}\left(\beta B_{2}-\kappa_{21}\right) .
\end{aligned}
$$

On the strengh of (3.8) and (3.6), $Y$ is negative in a small right neighborhood of 0 , and for all $\tau>0, B_{2}^{\prime}(\tau)<0$ and $B_{2}(\tau) \in(-\gamma, 0)$. Hence, under condition (3.11), $B_{2}^{\prime}(\tau)\left(\beta B_{2}(\tau)-\kappa_{21}\right)<0, \forall \tau>0$, and if $Y(\tau)$ 
tries to approach 0 from below, $Y^{\prime}(\tau)$ becomes negative. Thus, $B_{1}^{\prime}=Y$ must remain negative on $(0,+\infty)$.

b) The RHS of (3.4) is less than $-d_{0}+\alpha \gamma^{2} / 2$.

The next theorem justifies the use of the formal solution of ATSM.

Theorem 3.3. Let $g$ be bounded and continuous, and let

$$
d_{1}+\frac{\kappa_{11}^{2}}{2}-\kappa_{21} \gamma-\frac{\beta}{2} \gamma^{2}>0
$$

Then the expressions (1.1), (1.2) and (1.3) are the unique solution to the problem (1.4)-(1.5) in the class of continuous functions, which admit the bound

$$
|f(x, \tau)| \leq C \exp \left[\left(-\gamma x_{2}\right)_{+}\right] .
$$

Proof. The general scheme of the proof is described in Section 4, and the proof itself is given in Section 5 .

Remark 3.1. The bound (3.15), which specifies the class of functions among which the Feynman-Kac formula gives the unique solution, is natural because the bond price given by (1.6) satisfies this bound: see (3.6).

Remark 3.2. The LHS in the condition (3.14) summarizes the influence of different parameters of the process and the affine model of the short rate, which act in opposite directions. The main obstacle for the proof of the Feynman-Kac theorem (and the validity of this theorem) comes from the region in the state space where the short rate is unbounded from below. The larger the $d_{1}$, the smaller this region is, and the condition (3.14) provides the lower bound for $d_{1}$, which ensures the validity of the Feynman-Kac (the other parameters remaining fixed). In the $A_{0}(n)$ case, when the volatility is bounded and so trajectories of the process are not pushed too far too fast in the "negative" region, no additional condition is needed. In the $A_{1}(n)$ case, the entries of the volatility matrix are large in the region where $x_{1}$ is large, therefore the less time a trajectory of the process spends there, the smaller obstacle for the proof of the Feynman-Kac theorem (and the validity of this theorem) is. A large value of the mean-reverting entry $\kappa_{11}$ ensures that trajectories of the process do not remain far from the line $x_{1}=0$ for too long time, and the condition (3.14) provides the lower bound for $\kappa_{11}$, the other parameters remaining fixed.

The parameters $d_{2}, \beta$ and $\kappa_{22}$ act in opposite directions as well. The larger the $d_{2}$, the larger (the absolute value of) negative values of the short rate can be, and the larger the $\beta$, the farther a trajectory can be pushed into the "negative" region. The larger the $\kappa_{22}$ is, the faster a 
trajectory of the process will return to a neighborhood of a line $x_{2}=0$, where the short rate is bounded from below. If $\kappa_{21}=0$, then (3.14) provides an upper bound on $\beta\left(d_{2} / \kappa_{22}\right)^{2}$, which ensures the validity of the Feynman-Kac theorem. If $\kappa_{21} \neq 0$, the dynamics becomes more complex, and the term $\kappa_{21} \gamma$ takes this additional complexity into account.

Corollary 3.4. For the bond pricing problem, Theorem 3.3 is valid under condition

$$
d_{1}+\frac{\kappa_{11}^{2}}{2}-\kappa_{21} \gamma-\frac{\beta}{2} \gamma^{2} \geq 0
$$

Proof. Indeed, suppose that (3.16) holds with the equality. Then we can approximate $d_{1}$ by a sequence $\left\{d_{1, m}\right\}$ converging to $d_{1}$ from above. Each $d_{1, m}$ satisfies (3.14), and hence the conclusion of Theorem 3.3 holds. Set $r_{m}(x)=d_{0}+d_{1, m} x_{1}+d_{2} x_{2}$. By the Dominant Convergence Theorem, $f\left(r_{m}, g ; x, t\right) \rightarrow f(r, g ; x, t)$, point-wise, and the convergence $f_{0}\left(r_{m}, g ; x, t\right) \rightarrow f_{0}(r, g ; x, t)$ follows from the explicit formula for these functions and the theorem about the continuous dependence on parameters of the solution of a system of ODE of the first order.

3.2. Family $A_{1}(n)$. The state space is $\mathbf{R}_{+} \times \mathbf{R}^{n-1}$, the $r$ is given by (1.3) with $d_{j} \geq 0, j=1, \ldots, n$, and $d_{n}>0$, and the infinitesimal generator of the process is of the form

$$
L=\langle\theta-\kappa x, \partial\rangle+\frac{1}{2} x_{1} \partial_{1}^{2}+\frac{1}{2} \sum_{j, k=2}^{n}\left(\alpha_{j k}+x_{1} \beta_{j k}\right) \partial_{j} \partial_{k}
$$

where $\theta_{1}, \kappa_{j j}, j=1, \ldots, n$ are positive, $\kappa_{j l} \leq 0,1 \leq l<j, \kappa_{j k}=0, j<$ $k$, and $\alpha=\left[\alpha_{j k}\right]_{j, k=2}^{n}, \beta=\left[\beta_{j k}\right]_{j, k=2}^{n}$ are positive definite matrices (these restrictions can be relaxed). Without loss of generality, we may assume $\theta_{j}=0, j \geq 2$, when convenient.

Introduce vectors $d^{2}=\left(d_{2}, \ldots, d_{n}\right), \kappa^{21}=\left(\kappa_{21}, \ldots, \kappa_{n 1}\right)$, and set $\gamma=$ $\left(\kappa^{T}\right)^{-1} d$. The subsystem of the Riccati equations for $\mathcal{B}=\left(B_{2}, \ldots, B_{n}\right)$ enjoys the same properties as the subsystem for $B$ in the $A_{0}(n)$-model above, therefore the same proof as of Theorem 2.2 gives

Lemma 3.5. For $j=2, \ldots, n$, function $B_{j}$ is continuous and nonincreasing on $[0,+\infty)$, and $B_{j}(0)=0, B_{j}(+\infty)=-\gamma_{j}$.

It remains to study the monotonicity of $B_{1}$ and $C$. Introduce quadratic polynomials

$$
Q_{B}(y)=-d_{1}-\left\langle y, \kappa^{21}\right\rangle+\frac{1}{2} y^{T} \beta y, \quad y \in \mathbf{R}^{n-1},
$$


and

$$
Q_{C}(y)=-d_{0}+\frac{1}{2} y^{T} \alpha y, \quad y \in \mathbf{R}^{n-1} .
$$

The remaining two Riccati equations, for $B_{1}$ and $C$, are

$$
\begin{aligned}
& B^{\prime}(\tau)=-\kappa_{11} B_{1}(\tau)+\frac{1}{2} B_{1}^{2}(\tau)+Q_{B}(\mathcal{B}(\tau)), \\
& C^{\prime}(\tau)=\theta_{1} B_{1}(\tau)+Q_{C}(\mathcal{B}(\tau)) .
\end{aligned}
$$

Each of equations (3.18) and (3.19) is similar to equation (2.3) for $C$ in the $A_{0}(n)$-model, therefore the same proof as of Theorem 2.2 gives

Theorem 3.6. a) If $B_{1}$ is non-increasing on $[0,+\infty)$, then

$$
d_{1}>0 \text { or }\left\langle d^{2}, \kappa^{21}\right\rangle<0 \text {, }
$$

and

$$
Q_{B}\left(-\gamma_{2}, \ldots,-\gamma_{n}\right) \leq 0
$$

b) Let (3.20) hold, and

$$
Q_{B}(y) \leq 0, \quad \forall y \in\left\{-\gamma_{2}, 0\right\} \times \cdots \times\left\{-\gamma_{n}, 0\right\} .
$$

Then $B_{1}$ decreases on $[0,+\infty)$.

c) Let (3.20) and (3.22) hold, and

$$
Q_{C}(y) \leq 0, \quad \forall y \in\left\{-\gamma_{2}, 0\right\} \times \cdots \times\left\{-\gamma_{n}, 0\right\} .
$$

Then $C$ decreases on $[0,+\infty)$, and the bond price is a decreasing function of $(x, \tau)$ in the region $x>0, \tau>0$.

By using the family of transformations described in Dai and Singleton $(2000)$, it is possible to reduce any $A_{1}(n)$-model to a model with $\kappa$ satisfying

$$
\kappa_{j k}=0, \quad \forall k \neq j, k \geq 2, j \geq 1
$$

and we will formulate the theorem about the justification of the use of the Feynman-Kac theorem under this assumption.

Theorem 3.7. Let $g$ be bounded and continuous, let 3.24) hold, and let

$$
\frac{\kappa_{11}^{2}}{2}-Q_{B}(y)>0, \quad \forall y \in\left\{-\gamma_{2}, 0\right\} \times \cdots \times\left\{-\gamma_{n}, 0\right\} .
$$

Then the expressions (1.1), (1.2) and (1.3) are the unique solution to the problem (1.4)-(1.5) in the class of continuous functions, which admit the bound

$$
|f(x, \tau)| \leq C \exp \left[\sum_{j=2}^{n}\left(-\gamma_{j} x_{j}\right)_{+}\right]
$$

Proof. In Subsection 5.3. 
Remark 3.3. The bound (3.26), which specifies the class of functions among which the Feynman-Kac formula gives the unique solution, is natural because the bond price given by (1.6) satisfies this bound: see Lemma 3.5

Remark 3.4. In order to apply Theorem 3.7 to a particular model, the family of transformations described in Dai and Singleton (2000) should be used to obtain a model which satisfies (3.24). The condition (3.25) is a natural generalization of the condition (3.14) for the multi-factor case; the interpretation is essentially the same as in Remark 3.2.

Corollary 3.8. For the bond pricing problem, condition (3.25) in Theorem 3.7 can be replaced by a weaker one:

$$
\frac{\kappa_{11}^{2}}{2}-Q_{B}(y) \geq 0, \quad \forall y \in\left\{-\gamma_{2}, 0\right\} \times \cdots \times\left\{-\gamma_{n}, 0\right\} .
$$

3.3. Family $A_{2}(3)$. By using the family of transformations described in Dai and Singleton (2000), it is possible to reduce any three-factor ATSM with two factors of the CIR-type to a model of the form

$$
d X(t)=(\theta-\kappa X(t)) d t+\sqrt{\left[S_{j j}(t)\right]} d B(t),
$$

where $\theta \in \mathbf{R}^{3}$ is the vector with components $\theta_{3}=0$,

$$
\theta_{1}>0, \quad \theta_{2}>0
$$

and the entries of matrix $\kappa=\left[\kappa_{j l}\right]$ satisfy

$$
\begin{aligned}
& \kappa_{11}, \kappa_{22}, \kappa_{33}>0 \\
& \kappa_{21}, \kappa_{12} \leq 0 \leq 0 \\
& \kappa_{11} \kappa_{22}-\kappa_{12} \kappa_{21}>0 \\
& \kappa_{13}=\kappa_{23}=0 .
\end{aligned}
$$

Further,

$$
\begin{aligned}
& S_{j j}(t)=\beta_{j j} X_{j}(t), \quad j=1,2, \\
& S_{33}(t)=\alpha_{3}+\beta_{31} X_{1}(t)+\beta_{32} X_{2}(t),
\end{aligned}
$$

where

$$
\alpha_{3}, \beta_{11}, \beta_{22}>0, \quad \beta_{31}, \beta_{32} \geq 0,
$$

and finally, the short rate process is given by (1.3) with

$$
d_{0} \in \mathbf{R}, \quad d_{1}, d_{2} \geq 0, \quad d_{3}>0 .
$$

Notice that under conditions (3.29) and (3.30) - (3.33), any trajectory of the process $X$, which starts in the region $x_{1} \geq 0, x_{2} \geq 0$, remains in this region, a.s. 
The infinitesimal generator of the process is

$$
L=(\theta-\kappa x)^{T} \partial_{x}+\frac{1}{2} \sum_{j=1,2} \beta_{j j} x_{j} \partial_{j}^{2}+\frac{1}{2}\left(\alpha_{3}+\beta_{31} x_{1}+\beta_{32} x_{2}\right) \partial_{3}^{2},
$$

and therefore the system of Riccati equations is

$$
\begin{aligned}
& B_{1}^{\prime}=-\kappa_{11} B_{1}-\kappa_{21} B_{2}+\frac{\beta_{11}}{2} B_{1}^{2}-d_{1}-\kappa_{31} B_{3}+\frac{\beta_{31}}{2} B_{3}^{2} \\
& B_{2}^{\prime}=-\kappa_{12} B_{1}-\kappa_{22} B_{2}+\frac{\beta_{22}}{2} B_{2}^{2}-d_{2}-\kappa_{32} B_{3}+\frac{\beta_{32}}{2} B_{3}^{2} \\
& B_{3}^{\prime}=-\kappa_{33} B_{1}-d_{3} \\
& C^{\prime}=-d_{0}+\theta_{1} B_{1}+\theta_{2} B_{2}+\frac{\alpha_{3}}{2} B_{3}^{2} .
\end{aligned}
$$

From the initial condition

$$
B_{1}(0)=B_{2}(0)=B_{3}(0)=C(0)=0
$$

and (3.40) we can easily find $B_{3}$;

$$
B_{3}(\tau)=-\gamma\left(1-e^{-\kappa_{33} \tau}\right)
$$

where $\gamma=d_{3} / \kappa_{33}>0$. Clearly, $B_{3}$ decays on $[0,+\infty)$, and

$$
\begin{aligned}
B_{3}(+\infty) & =-\gamma, \\
B_{3}(\tau) & \in(-\gamma, 0), \quad \forall \tau>0, \\
B_{3}(\tau) & \sim-d_{3} \tau, \quad \text { as } \tau \rightarrow+0 .
\end{aligned}
$$

The monotonicity of $B_{j}, j=1,2$, implies additional restrictions on the parameters of the model. To formulate them, denote by $\tilde{\kappa}^{11}$ the inverse to the upper left $2 \times 2$ block $\kappa^{11}:=\left[\kappa_{j l}\right]_{j, l=1,2}$ of matrix $\kappa$,

$$
\tilde{\kappa}^{11}=\frac{1}{\kappa_{11} \kappa_{22}-\kappa_{12} \kappa_{21}}\left[\begin{array}{rr}
\kappa_{22} & -\kappa_{12} \\
-\kappa_{21} & \kappa_{11}
\end{array}\right]
$$

and set

$$
\left[\begin{array}{l}
\tilde{d}_{1} \\
\tilde{d}_{2}
\end{array}\right]=\tilde{\kappa}^{11}\left[\begin{array}{l}
d_{1} \\
d_{2}
\end{array}\right], \quad\left[\begin{array}{l}
\tilde{\kappa}_{31} \\
\tilde{\kappa}_{32}
\end{array}\right]=\tilde{\kappa}^{11}\left[\begin{array}{l}
\kappa_{31} \\
\kappa_{32}
\end{array}\right], \quad\left[\begin{array}{l}
\tilde{\beta}_{31} \\
\tilde{\beta}_{32}
\end{array}\right]=\tilde{\kappa}^{11}\left[\begin{array}{l}
\beta_{31} \\
\beta_{32}
\end{array}\right] .
$$

On the strength of (3.30)-(3.32), the diagonal (resp., off-diagonal) entries of $\tilde{\kappa}^{11}$ are positive (resp., non-positive), therefore

$$
\tilde{\beta}_{31} \geq 0, \quad \tilde{\beta}_{32} \geq 0,
$$

and

$$
\begin{aligned}
d_{1}, d_{2} \geq 0 & \Rightarrow \quad \tilde{d}_{1}, \tilde{d}_{2} \geq 0 \\
\kappa_{31}, \kappa_{32}<0 & \Rightarrow \quad \tilde{\kappa}_{31}, \tilde{\kappa}_{32}<0 .
\end{aligned}
$$


Theorem 3.9. Let $B_{j}, j=1,2$, be non-increasing on $[0,+\infty)$. Then the following conditions hold, for $j \neq l \in\{1,2\}$ :

$$
\begin{aligned}
& d_{j}>0 \quad \text { or } \quad d_{j}=0 \quad \text { and } \quad \kappa_{l j} d_{l}+\kappa_{3 j} d_{3} \leq 0 \text {; } \\
& \tilde{d}_{j}>0 \quad \text { or } \quad \tilde{d}_{j}=0 \quad \text { and } \quad \tilde{\kappa}_{3 j} \leq 0 \text {; } \\
& \tilde{d}_{j}-\tilde{\kappa}_{3 j} \gamma-\frac{\tilde{\beta}_{3 j}}{2} \gamma^{2} \geq 0 .
\end{aligned}
$$

Proof. a) If $d_{j}<0$, then (3.38)-(3.39) and (3.42) imply that $B_{j}^{\prime}(\tau)>0$ in a right neighborhood of 0 , contradiction. Hence, both $d_{j} \geq 0$. If both $d_{j}=0$, then from (3.38) - (3.39) and (3.46), both $B_{j}(\tau)=o(\tau)$, as $\tau \rightarrow+0$, and moreover,

$$
B_{j}^{\prime}(\tau) \sim \kappa_{3 j} d_{3} \tau, \quad \tau \rightarrow+0 .
$$

Hence, it is necessary that $\kappa_{3 j} \leq 0$. Finally, if $d_{1}=0$ but $d_{2}>0$, then $B_{2}(\tau) \sim-d_{2} \tau$, as $\tau \rightarrow+0$, hence,

$$
B_{1}^{\prime}(\tau) \sim\left(\kappa_{21} d_{2}+\kappa_{31} d_{3}\right) \tau, \quad \tau \rightarrow+0 .
$$

This excludes the case $\kappa_{21} d_{2}+\kappa_{31} d_{3}>0$, and finishes the proof of (3.50).

b) To prove (3.51)-(3.52), we apply $\tilde{\kappa}^{11}$ to subsystem (3.38)-(3.39); the result is

$$
\begin{aligned}
\tilde{\kappa}^{11}\left[\begin{array}{l}
B_{1}^{\prime} \\
B_{2}^{\prime}
\end{array}\right]= & -\left[\begin{array}{l}
B_{1} \\
B_{2}
\end{array}\right]+\frac{1}{2} \tilde{B}\left[\begin{array}{l}
B_{1}^{2} \\
B_{2}^{2}
\end{array}\right] \\
& -\left[\begin{array}{c}
\tilde{d}_{1} \\
\tilde{d}_{2}
\end{array}\right]-B_{3}\left[\begin{array}{c}
\tilde{\kappa}_{31} \\
\tilde{\kappa}_{32}
\end{array}\right]+\frac{1}{2} B_{3}^{2}\left[\begin{array}{c}
\tilde{\beta}_{31} \\
\tilde{\beta}_{32}
\end{array}\right],
\end{aligned}
$$

where

$$
\tilde{B}=\tilde{\kappa}^{11}\left[\begin{array}{ll}
\beta_{11} & 0 \\
0 & \beta_{22}
\end{array}\right] .
$$

Since $B_{1}^{\prime}$ and $B_{2}^{\prime}$ are non-positive, and the entries of $\tilde{\kappa}^{11}$ are nonnegative, the LHS in (3.53) is non-positive. Now by using (3.53) and arguing as in part a) above, we deduce (3.51).

If (3.52) fails, then from (3.44) we conclude that in a neighborhood of $+\infty$, one of the components of the RHS in (3.53) is positive, contradiction.

The next theorem gives sufficient conditions for the monotonicity of $B_{j}$ and $C$; they are more stringent than the necessary conditions in Theorem 3.9. 
Theorem 3.10. a) Let the following conditions hold, for $j=1,2$ :

$$
\begin{array}{r}
d_{j}>0 \quad \text { or } \quad d_{j}=0 \text { and } \kappa_{3 j}<0 ; \\
d_{j}-\kappa_{3 j} \gamma-\frac{\beta_{3 j}}{2} \gamma^{2} \geq 0 ; \\
\kappa_{3 j}+\beta_{3 j} \gamma \leq 0, \\
\beta_{3 j}>0 \text { or } \kappa_{3 j}<0 .
\end{array}
$$

Then $B_{1}$ and $B_{2}$ are decreasing on $[0,+\infty)$.

b) In addition, let

$$
d_{0} \geq \frac{\alpha_{3}}{2} \gamma^{2}
$$

Then $C$ decreases on $[0,+\infty)$, and the bond price is a decreasing function of $(x, \tau)$ in the region $x_{1}>0, x_{2}>0, \tau>0$.

Proof. a) First, we show that there exists $\tau_{0}>0$ such that for $j=1,2$,

$$
B_{j}(\tau)<0, \quad 0<\tau<\tau_{0}
$$

and

$$
B_{j}^{\prime}(\tau)<0, \quad 0<\tau<\tau_{0} .
$$

We use (3.38)-(3.39), (3.42) and (3.46). If $d_{1}$ and $d_{2}$ are positive, then $B_{j}^{\prime}(\tau) \sim-d_{j}$, as $\tau \rightarrow+0$, hence (3.60) hold, and (3.59) holds as well. If both $d_{j}=0$ but both $\kappa_{3 j}<0$, then $B_{j}^{\prime}(\tau) \sim \kappa_{3 j} d_{3} \tau$, as $\tau \rightarrow+0$, and (3.59) and (3.60) hold. Finally, if one of $d_{j}$, say, $d_{2}$, is positive, and the other, $d_{1}$, is 0 , then $B_{2}^{\prime}(\tau) \sim-d_{2}, B_{2}(\tau) \sim-d_{2} \tau$, as $\tau \rightarrow+0$, and

$$
B_{1}^{\prime}(\tau) \sim\left(\kappa_{21} d_{2}+\kappa_{31} d_{3}\right) \tau, \quad \tau \rightarrow+0 .
$$

Since $\kappa_{21} \leq 0, d_{2} \geq 0$ and $d_{3}>0$, we obtain (3.59)-(3.60).

Second, we show that (3.59) holds with $\tau_{0}=+\infty$. Under condition (3.54), the polynomials

$$
Q_{j}(y)=-d_{j}-\kappa_{3 j} y+\frac{\beta_{3 j}}{2} y^{2}
$$

are negative on $(-\gamma, 0)$, therefore in view of (3.45),

$$
-d_{j}+\kappa_{3 j} B_{3}(\tau)+\frac{\beta_{3 j}}{2} B_{3}(\tau)^{2}<0, \quad \forall \tau>0 .
$$

Suppose that as $\tau$ increases, $B_{j}(\tau), j=1,2$, start to approach 0 from below, simultaneously. Then from (3.38)-(3.39) and (3.61), at least one of $B_{j}^{\prime}(\tau)$ becomes negative before both $B_{j}(\tau), j=1,2$, reach 0 , contradiction. If $B_{1}(\tau)$ is approaching 0 but $B_{2}(\tau)$ is not, then eventually, on the strength of (3.38) and condition $\kappa_{21} \leq 0, B_{1}^{\prime}(\tau)$ becomes negative, contradiction. Thus, (3.59) holds on the whole half-axis. 
It remains to prove that $Y_{j}:=B_{j}^{\prime}$ are negative on the whole half-axis, $j=1,2$. We differentiate (3.38)- (3.39):

$$
\begin{aligned}
& Y_{1}^{\prime}=-\kappa_{11} Y_{1}-\kappa_{21} Y_{2}+\beta_{11} B_{1} Y_{1}-\kappa_{31} Y_{3}+\beta_{31} B_{3} Y_{3}, \\
& Y_{2}^{\prime}=-\kappa_{12} Y_{1}-\kappa_{22} Y_{2}+\beta_{22} B_{2} Y_{2}-\kappa_{32} Y_{3}+\beta_{32} B_{3} Y_{3},
\end{aligned}
$$

and rewrite (3.62)-(3.63) as

$$
\begin{aligned}
& Y_{1}^{\prime}=-\left(\kappa_{11}-\beta_{11} B_{1}\right) Y_{1}-\kappa_{21} Y_{2}+\left(\beta_{31} B_{3}-\kappa_{31}\right) Y_{3}, \\
& Y_{2}^{\prime}=-\kappa_{12} Y_{1}-\left(\kappa_{22}-\beta_{22} B_{2}\right) Y_{2}+\left(\beta_{32} B_{3}-\kappa_{32}\right) Y_{3} .
\end{aligned}
$$

Since $B_{j}$ are negative, $\kappa_{j j}-\beta_{j j} B_{j}>0, j=1,2$, and due to (3.45), (3.57) and (3.56), all the expressions in the brackets are positive. Since $Y_{3}$ is negative, $Y_{1}^{\prime}(\tau)$ and $Y_{2}^{\prime}(\tau)$ cannot approach 0 from below simultaneously: indeed, then eventually, the RHS's will become negative, contradiction. Since $-\kappa_{21} \geq 0$, we have $-\kappa_{21} Y_{2}(\tau) \leq 0$ where $Y_{2}(\tau)<0$, therefore by the same reasoning, $Y_{1}(\tau)$ cannot approach 0 from below while $Y_{2}(\tau)$ remains separated from 0 . By interchanging the indices 1 and 2 , we conclude that (3.60) holds on the whole half-axis.

b) Recall that $\theta_{1}$ and $\theta_{2}$ are positive, and apply (3.41), 3.45) and (3.59).

Theorem 3.11. Let $g$ be bounded and continuous, and let the following conditions hold: for $y=0, \gamma$,

$$
\begin{aligned}
& d_{1}+\frac{\kappa_{11}^{2}}{2 \beta_{11}}+\frac{\kappa_{21} \kappa_{22}}{\beta_{22}}-\kappa_{31} y-\frac{\beta_{31}}{2} y^{2}>0 \\
& d_{2}+\frac{\kappa_{22}^{2}}{2 \beta_{22}}+\frac{\kappa_{12} \kappa_{11}}{\beta_{11}}-\kappa_{32} y-\frac{\beta_{32}}{2} y^{2}>0 .
\end{aligned}
$$

Then the expressions (1.1), (1.2) and (1.3) are the unique solution to the problem (1.4)-(1.5) in the class of continuous functions, which admit the bound

$$
|f(x, \tau)| \leq C \exp \left[\left(-\gamma x_{3}\right)_{+}\right] .
$$

Proof. In Subsection 5.4,

Remark 3.5. The bound (3.66), which specifies the class of functions among which the Feynman-Kac formula gives the unique solution, is natural because the bond price given by (1.6) satisfies this bound: see (3.43).

Remark 3.6. The pair of conditions (3.64)-(3.65) is a natural generalization of the condition (3.14) in the $A_{1}(2)$ model; the interpretation is essentially the same as in Remark 3.2. 


\section{Justification of the use of the Feynman-KaC Formula}

4.1. General scheme. Step 1. We assume that $g$ is non-negative, bounded and sufficiently regular, so that in the case of a continuous $r$ bounded from below (not necessarily affine), problem (1.4)-(1.5) has a solution, $f_{0}(g, r ; \cdot, \cdot)$, in the class of bounded continuous functions, which is given by the stochastic expression (1.1): $f_{0}(g, r ; x, t)=$ $f(g, r ; x, t)$ for all $x$ and $t<T$. For affine diffusions, the coefficients of the infinitesimal generator of the process satisfy the global Lipschitz condition, and hence, the Feyman-Kac theorem is applicable. (The reduction to the case of more general $g$ is fairly standard).

Step 2. Fix $N \in \mathbf{R}$, and for the affine $r$, set $r_{N}(x)=\max \{r(x),-N\}$. Then $r_{N}$ is bounded from below, hence both $f\left(g, r_{N} ; x, t\right)$ and $f_{0}\left(g, r_{N} ; x, t\right)$ exist, and $f\left(g, r_{N} ; x, t\right)=f_{0}\left(g, r_{N} ; x, t\right)$ for all $x$ and $t<T$.

Step 3. By the Monotone Convergence Theorem,

$$
f\left(g, r_{N} ; x, t\right) \rightarrow f(g, r ; x, t) \text { as } N \rightarrow+\infty,
$$

point-wise, therefore it remains to show that

$$
f_{0}\left(g, r_{N} ; x, t\right) \rightarrow f_{0}(g, r ; x, t) \text { as } N \rightarrow+\infty,
$$

point-wise.

Step 4. We prove (4.1) by reducing to the case of a family of short rates, which is bounded from below uniformly in $N$. To this end, we take a non-negative function $\phi \in C^{\infty}\left(\mathbf{R}^{n}\right)$, and consider the representation

$$
\exp (-\phi(x)) L \exp (\phi(x))=L_{\phi}-\tilde{r}_{\phi}(x),
$$

where $L_{\phi}$ is a differential operator without the zero-order term, and $\tilde{r}_{\phi}$ is a function. In the presence of factors of the CIR-type, the infinitesimal generator may have non-trivial affine coefficients at the second order derivatives, therefore the choice of $\phi$ in the form of a quadratic polynomial, as in the proof of Theorem 2.3. is impossible except for some very special cases. One may try $\phi$ 's which are (approximately) positive homogenous of degree 1 in a neighborhood of infinity. The simplest version $\phi(x)=\langle A x, x\rangle /\langle x\rangle$, where $\langle x\rangle:=\left(1+\|x\|^{2}\right)^{1 / 2}$, is possible but it requires unnecessary strong restrictions on parameters of the model. It turns out that the construction of $\phi$ should be adjusted to each model. For the proof to work, the following general properties of $\phi$ are essential (they can be relaxed, though):

(i) there exist constants $c_{0}>0$ and $C$ such that

$$
\phi(x) \geq c_{0}|x|-C ;
$$


(ii) there exist constants $C_{0}>0, M, C_{1}$ such that $r_{\infty, \phi}:=r+\tilde{r}_{\phi}$ admits the following estimate:

$$
C_{0}|x|-M \leq r_{\infty, \phi}(x) \leq C_{1}|x|+M, \quad \forall x ;
$$

(iii) there exist constants $C_{2}$ and $\delta>0$ such that for all $x$ and $t \in$ $[0, T]$, the function $f_{\phi}(g, r ; x, t):=\exp (-\phi(x)) f_{0}(g, r ; x, t)$ satisfies an estimate

$$
\left|f_{\phi}(g, r ; x, t)\right| \leq C_{2} \exp (-\delta|x|)
$$

(iv) $L_{\phi}$ is sufficiently regular in the sense that for any $r^{0}$ which admits the bound (4.4), and a continuous $g^{0}(x)$ admitting the bound

$$
\left|g^{0}(x)\right| \leq C_{3} \exp (-\delta|x|),
$$

where $\delta>0$ and $C_{3}$ are independent of $x$, a continuous solution to the problem

$$
\begin{aligned}
\left(\partial_{t}+L_{\phi}-r^{0}\right) f(x, t) & =0, \quad 0 \leq t<T ; \\
f(x, T) & =g^{0}(x),
\end{aligned}
$$

which exponentially decays at infinity, exists and it is unique; call it $f^{\phi}\left(g^{0}, r^{0} ; x, t\right)$ (we add indices 0 , and use labels $r^{0}$ and $\left.g^{0}\right)$ in order to avoid the confusion with $g$ and $r$ in (1.4) $-(1.5)$ );

(v) as $N \rightarrow+\infty$,

$$
f^{\phi}\left(g^{0}, r_{N, \phi} ; x, t\right) \rightarrow f^{\phi}\left(g^{0}, r_{\infty, \phi} ; x, t\right),
$$

point-wise; here $r_{N, \phi}:=r_{N}+\tilde{r}_{\phi}$.

Notice that the construction of $\phi$ will depend on a small parameter $\epsilon \in(0,1)$, and the $r_{N, \phi}$ will satisfy a weak version of the global Lipschitz condition, with parameter, which simplifies the proof of the existence and uniqueness theorem in Section 5. We do not specify this condition here because it is a useful technical tool only, and it should be possible to prove the existence and uniqueness theorem under weaker conditions.

When the existence and uniqueness theorem is proved, the convergence (4.9) is typically not difficult to establish. For instance, if it can be shown that $L_{\phi}$ is the generator of a Markov process without killing, and the Feynman-Kac theorem is applicable, then (4.9) can be easily deduced from the Feynman-Kac formula and the Dominant Convergence Theorem. However, for the $L_{\phi}$, which we will construct below, there is no ready Feynman-Kac theorem available, and so (4.9) will be proved differently, by using the representation theorem for analytic semigroups. 
When the $\phi$ is constructed, and properties (i)-(v) are established, we can prove (4.1) as follows. For $N=1,2, \ldots, \infty$, set

$$
\begin{aligned}
g_{\phi}(x) & =\exp (-\phi(x)) g(x), \\
f_{\phi}\left(g, r_{N} ; x, t\right) & =\exp (-\phi(x)) f_{0}\left(g, r_{N} ; x, t\right),
\end{aligned}
$$

substitute $f_{0}\left(g, r_{N} ; x, t\right)=\exp (\phi(x)) f_{\phi}\left(g, r_{N} ; x, t\right)$ into (1.4)-(1.5), and multiply by $\exp (-\phi(x))$; we obtain that $f_{\phi}\left(g, r_{N} ; \cdot, \cdot\right)$ is a continuous solution to the problem

$$
\begin{aligned}
\left(\partial_{t}+L_{\phi}-r_{N, \phi}\right) f(x, t) & =0, \quad 0 \leq t<T \\
f(x, T) & =g_{\phi}(x)
\end{aligned}
$$

which exponentially decays at infinity. Clearly, $r_{N, \phi}$ satisfy (4.4) with the constants independent of $N=1,2, \ldots, \infty$; the RHS in (4.8) admits bound (4.6) since $g$ is bounded, and $\phi$ satisfies (4.3). By (iv), the continuous solution to problem (4.10)-(4.11), which exponentially decays at infinity, is unique, hence $f_{\phi}\left(g, r_{N} ; \cdot, \cdot\right)=f^{\phi}\left(g_{\phi}, r_{N, \phi} ; \cdot, \cdot\right)$, and by $(\mathrm{v})$, as $N \rightarrow+\infty, f^{\phi}\left(g_{\phi}, r_{N, \phi} ; \cdot, \cdot\right)$ converges to $f^{\phi}\left(g_{\phi}, r_{\infty, \phi} ; \cdot, \cdot\right)$, pointwise; (4.1) follows.

4.2. Outline of the verification of conditions (iv)-(v). Let $x^{\prime}$ be the group of the CIR-type variables, and $x^{\prime \prime}$ the group of the other variables; then $x=\left(x^{\prime}, x^{\prime \prime}\right)$. Let $\left(R_{+}\right)^{m} \times \mathbf{R}^{n-m}$ be the corresponding decomposition of the state space. We apply the standard approach: first, we write problem (4.10)-(4.11) in the form

$$
\begin{aligned}
F^{\prime}(\tau)+A F(\tau) & =0, \quad \tau>0 \\
F(+0) & =g^{0}
\end{aligned}
$$

where $A=-L_{\phi}+r^{0}$ is a (partial) differential operator on $\left(R_{+}\right)^{m} \times$ $\mathbf{R}^{n-m}$, and for each $\tau, F(\tau)$ is a function on $\left(R_{+}\right)^{m} \times \mathbf{R}^{n-m}$. Next, by using the Laplace transform w.r.t. $\tau$, we reduce problem (4.10)-(4.11) to the family of problems

$$
(\lambda+A) \hat{F}(\lambda)=g^{0},
$$

where $\hat{F}$ is the Laplace transform of $F$, and $\lambda$ belongs to a half-plane of the complex plane, of the form $\left\{\lambda \mid \Im \lambda>\lambda_{0}\right\}$. Then, by using the theory of degenerate elliptic operators with parameter, we show that if $\lambda_{0}$ is sufficiently large, then the operator $\lambda+A$ is invertible uniformly w.r.t. $\lambda$ in the half-plane $\Im \lambda \geq \lambda_{0}$. This proves the existence and uniqueness of the solution to the boundary problem (4.10)-(4.11) (that is, part (iv) of the general scheme) but in a wider class of functions since under this approach, (4.13) is satisfied in a weak sense (in the sense of the theory of generalized functions). To show that the solution 
satisfies (4.13) in the strong sense, and to prove the convergence in part (v), some additional effort is needed. We show that the representation theorem for analytic semigroups can be applied to $A$, and derive (4.9) from this representation. We need the following definition and theorem.

For $\sigma \in(0, \pi)$ and $\lambda_{0} \geq 0$, set $\Sigma_{\sigma, \lambda_{0}}=\left\{\lambda \in \mathbf{C}|| \lambda \mid \geq \lambda_{0}, \arg \lambda \in\right.$ $[-\sigma, \sigma]\}$. Let $A$ be an operator in the Banach space $\mathcal{B}$, and let there exist $\sigma \in(0, \pi), \lambda_{0} \geq 0$, and $C_{1}$ such that for $\lambda \in \Sigma_{\sigma, \lambda_{0}}, \lambda+A$ is invertible, and the resolvent satisfies the estimate

$$
\left\|(\lambda+A)^{-1}\right\| \leq C_{1}(1+|\lambda|)^{-1} .
$$

Then $A$ is called a weakly $\sigma$-positive operator ${ }^{3}$. If $C=0, A$ is called $\sigma$-positive. Let $\mathcal{L}_{\sigma, \lambda_{0}}=\partial \Sigma_{\sigma, \lambda_{0}}$ be a regular contour, with a parameterization $\lambda=\lambda(t)$ satisfying $\arg \lambda(t)= \pm \sigma$ for $t$ in a neighborhood of $\pm \infty$.

The following theorem is a special case of the representation theorem for the analytic semigroups (see Section IX.10 in Yosida (1964)).

Theorem 4.1. Let $A$ be an unbounded operator in the Banach space $\mathcal{B}$, and let there exist $\sigma \in(\pi / 2, \pi)$ and $C$ such that $A$ is weakly $\sigma$-positive. Then

a) for any $g^{0} \in \mathcal{B}$, and any $\tau>0$, the following integral is well-defined

$$
\exp (-\tau A) g^{0}=(2 \pi i)^{-1} \int_{\mathcal{L}_{\sigma, \lambda_{0}}} e^{\tau \lambda}(\lambda+A)^{-1} g^{0} d \lambda
$$

b) (4.16) defines a strongly continuous semigroup $\left\{T_{\tau}\right\}_{\tau \geq 0}$ in $\mathcal{B}$ by $T_{0}=$ $I, T_{\tau}=\exp (-\tau A), \tau>0$, and

c) for any $g^{0} \in \mathcal{B}, F(\tau)=\exp (-\tau A) g^{0}$ is a strongly continuous solution to the problem (4.12)-4.13).

As $\mathcal{B}$, we take $L_{2}\left(\left(R_{+}\right)^{m} \times \mathbf{R}^{n-m}\right)$, and we show that the conditions of Theorem 4.1 are satisfied. For a function $h$, denote by $h(\cdot)$ or simply $h$ the-multiplication-by- $h$-operator. We will be able to prove that if $\rho>0$ is sufficiently small then

$$
\left\|\exp \left(\rho\langle\cdot\rangle^{\prime \prime}\right)\left(\lambda+r^{0}(\cdot)\right)(\lambda+A)^{-1} \exp \left(-\rho\langle\cdot\rangle^{\prime \prime}\right)\right\| \leq C_{1},
$$

for all $\lambda \in \Sigma_{\sigma, \lambda_{0}}$. By using the representation (4.16) and estimate (4.17), we can prove (4.9) as follows. Set $r^{0}=r_{\infty, \phi}, A=-L_{\phi}+r_{\infty, \phi}$, and let $F$ be the solution to (4.12)-(4.13). Let $F_{(N)}$ be the solution of the problem (4.12)-(4.13) with $A_{(N)}=-L_{\phi}+r_{N, \phi}$ instead of $A$. We show that $A$ and each $A_{(N)}$ satisfies (4.15) uniformly in $N$, with the

\footnotetext{
${ }^{3}$ Usually, the label $\theta$ is used instead of $\sigma$; unfortunately, $\theta$ is already occupied as the standard notation in ATSM's
} 
same $\Sigma_{\sigma, \lambda_{0}}$, and therefore the representation (4.16) holds for $F_{(N)}$ with $A_{(N)}$ instead of $A$. Notice that

$$
\begin{aligned}
\left(\lambda+A_{(N)}\right)^{-1} g^{0}-(\lambda+A)^{-1} g^{0} & =\left(\lambda+A_{(N)}\right)^{-1}\left(r_{\infty, \phi}-r_{N, \phi}\right)(\lambda+A)^{-1} g^{0} \\
& =\left(\lambda+A_{(N)}\right)^{-1} b_{N, \lambda} C_{\lambda} g^{1}
\end{aligned}
$$

where

$$
\begin{gathered}
b_{N, \lambda}:=\left(r_{\infty, \phi}-r_{N, \phi}\right) \exp \left(-\rho\langle\cdot\rangle^{\prime \prime}\right)\left(\lambda+r_{\infty, \phi}\right)^{-1} \\
C_{\lambda}=\exp \left(\rho\langle\cdot\rangle^{\prime \prime}\right)\left(\lambda+r_{\infty, \phi}(\cdot)\right)(\lambda+A)^{-1} \exp \left(-\rho\langle\cdot\rangle^{\prime \prime}\right),
\end{gathered}
$$

and $g^{1}:=\exp \left(\rho\langle\cdot\rangle^{\prime \prime}\right) g^{0}$ is a continuous function which decays at infinity, if $\rho<\delta$. It is easily seen that

$$
\lim _{N \rightarrow+\infty} \sup _{\lambda, x}\left|b_{N, \lambda}(x)\right|=0,
$$

therefore the norm of the-multiplication-by- $b_{N, \lambda}$ operator tends to zero as $N \rightarrow+\infty$, uniformly in $\lambda \in \mathcal{L}_{\sigma, \lambda_{0}}$; the norm of $C_{\lambda}$ is uniformly bounded w.r.t. $\lambda \in \mathcal{L}_{\sigma, \lambda_{0}}$ on the strength of (4.17). Hence,

$$
\begin{aligned}
F_{(N)}(\tau)-F(\tau) & =\exp \left(-\tau A_{(N)}\right) g^{0}-\exp (-\tau A) g^{0} \\
& =(2 \pi i)^{-1} \int_{\mathcal{L}_{\sigma, \lambda_{0}}} e^{\tau \lambda}\left(\left(\lambda+A_{(N)}\right)^{-1}-(\lambda+A)^{-1}\right) g^{0} d \lambda \\
& =(2 \pi i)^{-1} \int_{\mathcal{L}_{\sigma, \lambda_{0}}} e^{\tau \lambda}\left(\lambda+A_{(N)}\right)^{-1} b_{N, \lambda} C_{\lambda} g^{1} d \lambda
\end{aligned}
$$

vanishes as $N \rightarrow+\infty$, and (4.9) is proved.

The regularity theorem for locally elliptic operators guarantees that the solutions $f_{(N)}(x, \tau)=F_{(N)}(x)$ are not only in $L_{2}\left(\left(\mathbf{R}_{+}\right)^{m} \times \mathbf{R}^{n-m}\right)$ but continuous as well, and they decay at the infinity. Hence, the convergence in $C\left(\mathbf{R}_{+} ; L_{2}\left(\left(\mathbf{R}_{+}\right)^{m} \times \mathbf{R}^{n-m}\right)\right.$, the space of continuous vectorfunctions with values in $L_{2}\left(\left(\mathbf{R}_{+}\right)^{m} \times \mathbf{R}^{n-m}\right)$, implies the pointwise convergence of $f_{(N)}(x, \tau)$ to $f_{(\infty)}(x, \tau)$.

In Section [5, the construction of $\phi$ and the proof of the existence and uniqueness theorem and bound (4.17) will be provided for the family $A_{1}(2)$, and then for families $A_{1}(n), n \geq 2$, when the construction of $\phi$ becomes more involved. In Subsection 5.4, the modifications of the proof for the family $A_{2}(3)$ are outlined, and the proof for other families $A_{n}(m)$ is essentially the same.

\section{Proofs of sufficient Conditions for the Feynman-KaC}

THEOREM

\subsection{Proof of Theorem [3.3, part I: construction of $\phi$ and veri-} fication of conditions (4.2) $-(4.5)$. Fix any positive $\gamma_{+}$and

$$
\gamma_{-}<-\gamma \text {. }
$$


Next, take a non-decreasing function $\chi \in C^{\infty}(\mathbf{R})$ such that

$$
\begin{gathered}
\chi(y)=\gamma_{-}, \quad y<-1 \\
\chi(y)=\gamma_{+}, \quad y>0
\end{gathered}
$$

and

$$
\gamma_{-} \leq \chi(y) \leq \gamma_{+}, \quad \forall y \in \mathbf{R}
$$

Then, for any $\epsilon \in(0,1)$, construct functions $\chi_{\epsilon}(y)=\chi(\epsilon y)$ and

$$
\psi_{\epsilon}(y)=\int_{0}^{y} \chi_{\epsilon}(s) d s
$$

and finally, set

$$
\phi_{\epsilon}(x)=\kappa_{11} x_{1}+\psi_{\epsilon}\left(x_{2}\right) .
$$

Clearly, $\phi_{\epsilon}$ satisfies (4.3). Set $\delta:=\max \left\{\gamma_{+}, \kappa_{11},-\gamma-\gamma_{-}\right\}$; it is positive since $\kappa_{11}>0, \gamma_{+}>0$ and (5.1) holds.

Lemma 5.1. For any $\epsilon \in(0,1)$, there exists $C_{\epsilon}$ such that for all $x_{1}>0$ and $x_{2} \in \mathbf{R}$,

$$
\exp \left(-\phi_{\epsilon}\left(x_{2}\right)\right)\left|f_{0}(r, g ; x, t)\right| \leq C_{\epsilon} \exp \left(-\delta\left(x_{1}+\left|x_{2}\right|\right)\right) .
$$

In particular, (4.5) holds.

Proof. From the explicit solution to the bond pricing problem, we know that $f_{0}(r, 1 ; x, t)$ admits the bound (3.15). Since $g$ is bounded, $f_{0}(r, g ; x, t)$ admits the same bounds (with a different $C$, perhaps). Hence, on the set $\left\{x \mid x_{1}>0, x_{2}>-1 / \epsilon\right\}$, function $f_{0}(r, g ; \cdot, \cdot)$ is bounded, and since $\phi_{\epsilon}$ is bounded from below by a linear function with positive coefficients, $\kappa_{11} x_{1}+\gamma_{+} x_{2}$, we conclude that estimate (5.5) holds on this set. On the set $\left\{x \mid x_{1}>0, x_{2}<-1 / \epsilon\right\}$, function $f_{0}(r, g ; \cdot, \cdot)$ is bounded from above by an exponential function of the form $C \exp \left(-\gamma x_{2}\right)$, and $\phi_{\epsilon}$ is bounded from below by an affine function $\kappa_{11} x_{1}+\gamma_{-} x_{2}+c$, where

$$
c=\epsilon^{-1} \int_{0}^{-1} \chi(y) d y .
$$

In view of our choice (5.1), estimate (5.5) holds on this set as well.

Now we can check that for any sufficiently small positive $\gamma_{+}$and $\epsilon \in(0,1)$, and $\gamma_{-}$in a sufficiently small left vicinity of $-\gamma$, the function $\phi_{\epsilon}$ satisfies conditions (4.2) and (4.4). The dependence on $\epsilon$ will also be used to check conditions (iv)-(v) of the general scheme: it is convenient to use the dependence on a small parameter, and prove the existence and uniqueness of the solution of the boundary problem (4.7)-(4.8) for sufficiently small $\epsilon>0$. 
We have

$$
\begin{aligned}
\exp \left(-\phi_{\epsilon}(x)\right) L \exp \left(\phi_{\epsilon}(x)\right)= & \left(\theta_{1}-\kappa_{11} x_{1}\right)\left(\partial_{1}+\kappa_{11}\right) \\
& -\left(\kappa_{21} x_{1}+\kappa_{22} x_{2}\right)\left(\partial_{2}+\chi_{\epsilon}\left(x_{2}\right)\right) \\
& +\frac{1}{2} x_{1}\left(\partial_{1}+\kappa_{11}\right)^{2}+\frac{\alpha+\beta x_{1}}{2}\left(\partial_{2}+\chi_{\epsilon}\left(x_{2}\right)\right)^{2},
\end{aligned}
$$

therefore

$$
\begin{aligned}
L_{\phi}= & \theta_{1} \partial_{1}+\frac{1}{2} x_{1} \partial_{1}^{2} \\
& +\left[-\kappa_{21} x_{1}-\kappa_{22} x_{2}+\left(\alpha+\beta x_{1}\right) \chi_{\epsilon}\left(x_{2}\right)\right] \partial_{2}+\frac{\alpha+\beta x_{1}}{2} \partial_{2}^{2}
\end{aligned}
$$

and

$$
\begin{aligned}
r_{\infty, \phi}(x)= & d_{0}-\theta_{1} \kappa_{11}-\frac{\alpha}{2}\left(\epsilon \chi_{\epsilon}^{\prime}\left(x_{2}\right)+\chi_{\epsilon}\left(x_{2}\right)^{2}\right) \\
& +\left[d_{1}+\frac{\kappa_{11}^{2}}{2}+\kappa_{21} \chi_{\epsilon}\left(x_{2}\right)-\frac{\beta}{2}\left(\epsilon \chi_{\epsilon}^{\prime}\left(x_{2}\right)+\chi_{\epsilon}\left(x_{2}\right)^{2}\right)\right] x_{1} \\
& +\left[d_{2}+\kappa_{22} \chi_{\epsilon}\left(x_{2}\right)\right] x_{2} .
\end{aligned}
$$

Here $\chi_{\epsilon}^{\prime}(y):=\left(\chi^{\prime}\right)_{\epsilon}(y):=\chi^{\prime}(\epsilon y)$.

Lemma 5.2. Let 3.14 hold. Then there exists $\epsilon_{0}>0, \gamma_{+}>0$ and $\gamma_{-}<-\gamma$ such that for all $\epsilon \in\left(0, \epsilon_{0}\right)$, estimate (4.4) holds with constants $C_{0}, C_{1}$ independent of $\epsilon \in\left(0, \epsilon_{0}\right)$, and $M=M_{0} \epsilon^{-1}$, where $M_{0}$ is also independent of $\epsilon$.

Proof. In view of (5.4), the first three terms on the RHS of (5.7) are bounded, and

$$
\sup \left|\epsilon \chi_{\epsilon}^{\prime}\left(x_{2}\right)\right| \rightarrow 0, \quad \epsilon \rightarrow 0,
$$

therefore it suffices to show that $\gamma_{+}>0$ and $\gamma_{-}<-\gamma$ can be chosen so that

1) $d_{1}+\kappa_{11}^{2} / 2+\kappa_{21} \chi_{\epsilon}\left(x_{2}\right)-\beta \chi_{\epsilon}\left(x_{2}\right)^{2} / 2$ is positive and bounded away from zero, uniformly in $x_{2}$ and $\epsilon>0$;

2) $d_{2}+\kappa_{22} \chi_{\epsilon}\left(x_{2}\right)$ is positive and bounded away from 0 , uniformly in $x_{2}>0$ and $\epsilon>0$

3) $d_{2}+\kappa_{22} \chi_{\epsilon}\left(x_{2}\right)$ is negative and bounded away from 0 , uniformly in $x_{2}<-1 / \epsilon$ and $\epsilon>0$.

Now, due to (5.2), 3) is $d_{2}+\kappa_{22} \gamma_{-}<0$, which is equivalent to (5.1), and due to (5.3), 2) reduces to $d_{2}+\kappa_{22} \gamma_{+}>0$, which is trivial. On the strength of (5.4), 1) is equivalent to the condition: the polynomial

$$
y \mapsto Q(y):=d_{1}+\frac{\kappa_{11}^{2}}{2}+\kappa_{21} y-\frac{\beta}{2} y^{2}
$$


is positive on $\left[\gamma_{-}, \gamma_{+}\right]$. Since $\beta>0$, it suffices to choose $\gamma_{ \pm}$so that $Q\left(\gamma_{ \pm}\right)>0$. If $\gamma_{+}>0$ is sufficiently small, then $Q\left(\gamma_{+}\right)$is positive since $Q(0)=d_{1}+\kappa_{11}^{2} / 2>0$ is, and if $Q(y)$ is positive at $y=-\gamma$ (which is condition (3.14) $)$, then it is positive for $\gamma_{-}$in a sufficiently small neighborhood of $-\gamma$.

\subsection{Proof of Theorem 3.3, part II: verification of conditions} (iv) $-(\mathbf{v})$ of the general scheme. When $\chi$ in the definition of $\phi_{\epsilon}$ is fixed, the $L_{\phi}$ depends on $\epsilon$ only, and to stress the dependence on $\epsilon$, in this subsection, we write $L_{\epsilon}$ instead of $L_{\phi}$. In the proofs of the existence and the uniqueness theorem for the solution of problem (4.10)-(4.11) and estimates (4.15) and (4.17), we use an additional property of $r^{0}$, which was omitted from the general scheme in Subsection 4.1 as too technical (and by no means necessary), and which holds for $r_{\infty, \phi}$ and $r_{N, \phi}$. Fix $\omega \in(0,1 / 2)$. We denote by $r_{\epsilon}$ any function (in fact, a family of functions, parametrized by $\epsilon \in(0,1)$ ), which admits bound (4.4) with the constants $C_{1}>0, C_{2}$ and $M=M_{0} \epsilon^{-1}$, where $C_{0}, C_{1}, M_{0}$ are independent of $\epsilon \in(0,1)$; in addition, $r_{\epsilon}$ must satisfy the following Lipschitz condition with weight:

there exists a constant $M_{1}>0$ independent of $\epsilon \in(0,1)$ and such that for any $x, y \in \mathbf{R}_{+} \times \mathbf{R}$ satisfying $\left|x_{j}-y_{j}\right| \leq\left(\left|x_{j}\right|+\epsilon^{-1}\right)^{\omega}, j=1,2$,

$$
\left|r_{\epsilon}(x)-r_{\epsilon}(y)\right| \leq M_{1}\left(|x|+\epsilon^{-1}\right) \epsilon^{1-\omega} .
$$

Lemma 5.3. Functions $r_{\infty, \phi}$ and $r_{N, \phi}$ satisfy (4.4) and (5.8), uniformly in $N$ and $\epsilon \in(0,1)$.

Proof. Each of the functions $r_{\infty, \phi}$ and $r_{N, \phi}$ is the sum of a linear function $r$ or a piece-wise linear function $r_{N}$, for which (5.8) is evident since $\omega \in(0,1 / 2)$, and the function $\tilde{r}_{\phi}$ of the form

$$
\tilde{r}_{\phi}(x)=b_{1, \epsilon}\left(x_{2}\right) x_{1}+b_{2, \epsilon}\left(x_{2}\right)+b_{0, \epsilon},
$$

where $b_{0, \epsilon}$ is bounded (hence, satisfies (5.8)), and $b_{j, \epsilon}, j=1,2$, are constant outside the set $x_{2} \in[-1 / \epsilon, 0]$, and have derivatives of order $\epsilon$ on this set. By applying the Lagrange theorem, we obtain (5.8) for $b_{1, \epsilon}\left(x_{2}\right) x_{1}+b_{2, \epsilon}\left(x_{2}\right)$. Thus, (5.8) has been proved.

The proof of bound (4.4) in Subsection 5.1] is applicable to any $r_{\epsilon}$, and the constants in (4.4) can be chosen the same for all $\epsilon \in(0,1)$.

The $-L_{\epsilon}$ is an elliptic operator in the half-space $x_{1}>0$, which degenerates at the boundary $x_{1}=0$, of the form

$$
-L_{\epsilon}=-\theta_{1} \partial_{1}-\frac{1}{2} x_{1} \partial_{1}^{2}+\mu_{\epsilon}(x) \partial_{2}-\frac{\alpha+\beta x_{1}}{2} \partial_{2}^{2},
$$


where $\theta_{1}, \alpha, \beta$ are positive, and $\mu_{\epsilon}$ is an affine function of $x_{1}$

$$
\mu_{\epsilon}(x)=\mu_{\epsilon, 0}\left(x_{2}\right)+x_{1} \mu_{\epsilon, 1}\left(x_{2}\right),
$$

whose coefficients are uniformly bounded, constant outside the segment $[-1 / \epsilon, 0]$, and satisfy the global Lipschitz condition with parameter:

$$
\left|\mu_{\epsilon, j}(y)-\mu_{\epsilon, j}(z)\right| \leq C \epsilon|y-z|,
$$

where $C$ is independent of $\epsilon \in(0,1)$.

Theorem 5.4. Let $-L_{\epsilon}$ be an operator of the form (5.9), where $\theta_{1}, \alpha, \beta$ are positive, and the real-valued function $\mu_{\epsilon}$ satisfies (5.10) and (5.11). Let $r_{\epsilon}$ satisfy (4.4) and (5.8), with constants $C, C_{0}, C_{1}, M_{0}$ independent of $\epsilon \in(0,1)$.

Then there exist $\epsilon_{0}>0$ such that for all $\epsilon \in\left(0, \epsilon_{0}\right)$

a) for any $T>0$ and any continuous $g^{0}$, which exponentially decays at infinity, the problem

$$
\begin{aligned}
\left(\partial_{\tau}-L_{\epsilon}+r_{\epsilon}\right) f(x, \tau) & =0, \quad \tau \in(0, T), \\
f(x, \tau) & =g^{0}
\end{aligned}
$$

has the unique continuous solution, which exponentially decays at infinity;

b) set $\lambda_{0}=2 M_{0} \epsilon^{-1}, \sigma=\pi / 2+\epsilon$; then there exists $C_{1}$ and $\rho_{0}>0$ such that if $|\rho| \leq \rho_{0}$ then for all $\epsilon \in\left(0, \epsilon_{0}\right)$ and $\lambda \in \Sigma_{\sigma, \lambda_{0}}$.

$$
\left\|\exp \left(\rho\langle\cdot\rangle^{\prime \prime}\right)\left(\lambda+r^{0}(\cdot)\right)\left(\lambda-L_{\epsilon}+r_{\epsilon}\right)^{-1} \exp \left(-\rho\langle\cdot\rangle^{\prime \prime}\right)\right\| \leq C_{1} .
$$

Proof. a) Instead of the problem on a strip $\tau \in[0, T]$, we can consider the problem on $\tau \geq 0$, and look for the solution in the class of continuous functions which admit a bound

$$
|f(x, \tau)| \leq C \exp \left[-\delta_{1}|x|+\tau\left(\lambda_{0}-\delta_{1}\right)\right]
$$

for some $\delta_{1}>0$. By using the Laplace transform, we can reduce the existence and uniqueness theorem to the uniform invertibility of the family $\lambda-L_{\epsilon}+r_{\epsilon}$, on the line $\Re \lambda=\lambda_{0}$; the uniform invertibility follows from part b). Notice that in order to prove that the solution satisfies the boundary condition (5.13) in the strong sense, the invertibility for $\lambda \in \Sigma_{\sigma, \lambda_{0}}$ and estimate (4.15) are needed.

b) will be proved in the appendix. Notice that both (4.15) and (4.17) are implied by (5.14). 
5.3. Proof of Theorem 3.7. For $j=2, \ldots, n$, fix $\gamma_{+, j}>0$ and

$$
\gamma_{-, j}<-\gamma_{j}
$$

Next, take a non-decreasing function $\chi_{j} \in C^{\infty}(\mathbf{R})$ such that

$$
\begin{gathered}
\chi_{j}(y)=\gamma_{-, j}, \quad y<-1 \\
\chi_{j}(y)=\gamma_{+, j}, \quad y>0
\end{gathered}
$$

and

$$
\gamma_{-, j} \leq \chi_{j}(y) \leq \gamma_{+, j}, \quad \forall y \in \mathbf{R}
$$

Then, for any $\epsilon \in(0,1)$, construct functions $\chi_{j, \epsilon}(y)=\chi_{j}(\epsilon y)$ and

$$
\psi_{j, \epsilon}(y)=\int_{0}^{y} \chi_{j, \epsilon}(s) d s
$$

and finally, set

$$
\phi_{\epsilon}(x)=\kappa_{11} x_{1}+\sum_{j=2}^{n} \psi_{j, \epsilon}\left(x_{j}\right)
$$

Clearly, it satisfies (4.3). Set

$$
\delta:=\max \left\{\kappa_{11}, \max _{j \geq 2} \gamma_{+, j}, \max _{j \geq 2}\left\{-\gamma_{j}-\gamma_{-, j}\right\}\right\} ;
$$

it is positive since $\kappa_{11}>0, \gamma_{+, j}>0$ and (5.16) holds.

Lemma 5.5. For any $\epsilon \in(0,1)$, there exists $C_{\epsilon}$ such that for all $x_{1}>0$ and $x_{2} \in \mathbf{R}$,

$$
\exp \left(-\phi_{\epsilon}\left(x^{\prime \prime}\right)\right)\left|f_{0}(r, g ; x, t)\right| \leq C_{\epsilon} \exp \left(-\delta\left(x_{1}+\left|x^{\prime \prime}\right|\right)\right)
$$

In particular, 4.5) holds.

The proof of this lemma is an evident modification of the proof of Lemma 5.5 in the case $n=2$, and the rest of the proof of Theorem 3.3 is a straightforward modification of constructions and arguments in Subsection 5.1 and Subsection 5.2 .

5.4. Proof of Theorem 3.11. Fix $\gamma_{-}<-\gamma$ and $\gamma_{+}>0, l_{1}, l_{2} \in \mathbf{R}$, and for $\epsilon>0$, construct $\chi_{\epsilon}$ and $\psi_{\epsilon}$ as in Section 5. After that, define

$$
\phi_{\epsilon}(x)=l_{1} x_{1}+l_{2} x_{2}+\psi_{\epsilon}\left(x_{3}\right) \text {. }
$$

Set

$$
\theta^{1}=\left[\begin{array}{l}
\theta_{1} \\
\theta_{2}
\end{array}\right], \quad \partial^{1}=\left[\begin{array}{l}
\partial_{1} \\
\partial_{2}
\end{array}\right] \quad x^{1}=\left[\begin{array}{l}
x_{1} \\
x_{2}
\end{array}\right]
$$


We have

$$
\begin{aligned}
\exp \left(-\phi_{\epsilon}(x)\right) L \exp \left(\phi_{\epsilon}(x)\right)= & \left\langle\theta^{1}-\kappa^{11} x^{1}, \partial^{1}+l^{1}\right\rangle \\
& -\left(\kappa_{31} x_{1}+\kappa_{32} x_{2}+\kappa_{33} x_{3}\right)\left(\partial_{3}+\chi_{\epsilon}\left(x_{3}\right)\right) \\
& +\frac{\beta_{11}}{2} x_{1}\left(\partial_{1}+l_{1}\right)^{2}+\frac{\beta_{22}}{2} x_{2}\left(\partial_{2}+l_{2}\right)^{2} \\
& +\frac{1}{2}\left(\alpha_{2}+\beta_{31} x_{1}+\beta_{32} x_{2}\right)\left(\partial_{3}+\chi_{\epsilon}\left(x_{3}\right)\right)^{2},
\end{aligned}
$$

therefore

$$
\begin{aligned}
L_{\phi}= & \left\langle\theta^{1}, \partial^{1}\right\rangle+\sum_{j=1,2} \frac{\beta_{j j}}{2} x_{j} \partial_{j}^{2}+\frac{1}{2}\left(\alpha_{3}+\beta_{31} x_{1}+\beta_{32} x_{2}\right) \partial_{3}^{2} \\
& +\left(\left(\beta_{11} l_{1}-\kappa_{11}\right) x_{1}-\kappa_{12} x_{2}\right) \partial_{1}+\left(\left(\beta_{22} l_{2}-\kappa_{22}\right) x_{2}-\kappa_{21} x_{1}\right) \partial_{2} \\
& +\left[-\kappa_{31} x_{1}-\kappa_{32} x_{2}-\kappa_{33} x_{3}+\left(\alpha_{3}+\beta_{31} x_{1}+\beta_{32} x_{2}\right) \chi_{\epsilon}\right] \partial_{3},
\end{aligned}
$$

and by denoting the columns of the matrix $\kappa^{11}$ as $\kappa^{j}, j=1,2$,

$$
\begin{aligned}
r_{\infty, \phi}(x)= & d_{0}-\left\langle\theta^{1}, l^{1}\right\rangle-\frac{\alpha_{3}}{2}\left(\epsilon \chi_{\epsilon}^{\prime}\left(x_{3}\right)+\chi_{\epsilon}\left(x_{3}\right)^{2}\right) \\
& +\left[d_{1}+\left\langle\kappa^{1}, l\right\rangle-\frac{\beta_{11} l_{1}^{2}}{2}+\kappa_{31} \chi_{\epsilon}\left(x_{3}\right)-\frac{\beta_{31}}{2}\left(\epsilon \chi_{\epsilon}^{\prime}\left(x_{3}\right)+\chi_{\epsilon}\left(x_{3}\right)^{2}\right)\right] x_{1} \\
& +\left[d_{2}+\left\langle\kappa^{2}, l\right\rangle-\frac{\beta_{22} l_{2}^{2}}{2}+\kappa_{32} \chi_{\epsilon}\left(x_{3}\right)-\frac{\beta_{32}}{2}\left(\epsilon \chi_{\epsilon}^{\prime}\left(x_{3}\right)+\chi_{\epsilon}\left(x_{3}\right)^{2}\right)\right] x_{2} \\
& +\left[d_{3}+\kappa_{33} \chi_{\epsilon}\left(x_{3}\right)\right] x_{3} .
\end{aligned}
$$

Now it is clear what the optimal choice of $l_{1}$ and $l_{2}$ is. Indeed, it is necessary that in the formula for $L_{\phi}$, the coefficients at $\partial_{j}, j=1,2$, must be non-negative, hence $l_{j} \geq \kappa_{j j} / \beta_{j j}$. On the other hand, in the formula for $r_{\infty, \phi}$, it is better to have the coefficients at $x_{j}, j=1,2$, as large as possible. Equivalently,

$$
\kappa_{11} l_{1}-\frac{\beta_{11} l_{1}^{2}}{2}+\kappa_{21} l_{2} \text { and } \kappa_{22} l_{2}-\frac{\beta_{22} l_{2}^{2}}{2}+\kappa_{12} l_{1}
$$

should be as large as possible. But for $j=1,2, \kappa_{j j} l_{j}-\beta_{j j} l_{j}^{2} / 2$ attains its maximum at $l_{j}=\kappa_{j j} / \beta_{j j}$, and since $\kappa_{21}$ is negative, $\kappa_{21} l_{2}$ is attains its maximum on $\left[\kappa_{22} / \beta_{22},+\infty\right)$ at $l_{2}=\kappa_{22} / \beta_{22}$. Hence, the optimal choice is $l_{j}=\kappa_{j j} / \beta_{j j}, j=1,2$, and with this choice, the coefficients at $x_{j}, j=1,2$, become

$$
d_{1}+\frac{\kappa_{11}}{2 \beta_{11}}+\frac{\kappa_{21} \kappa_{22}}{\beta_{22}}+\kappa_{31} \chi_{\epsilon}\left(x_{3}\right)-\frac{\beta_{31}}{2}\left(\epsilon \chi_{\epsilon}^{\prime}\left(x_{3}\right)+\chi_{\epsilon}\left(x_{3}\right)^{2}\right)
$$

and

$$
d_{2}+\frac{\kappa_{22}}{2 \beta_{22}}+\frac{\kappa_{12} \kappa_{11}}{\beta_{11}}+\kappa_{32} \chi_{\epsilon}\left(x_{3}\right)-\frac{\beta_{32}}{2}\left(\epsilon \chi_{\epsilon}^{\prime}\left(x_{3}\right)+\chi_{\epsilon}\left(x_{3}\right)^{2}\right) .
$$


If $\epsilon>0$ and $\gamma_{+}>0$ are sufficiently small, and $\gamma_{-}<-\gamma$ is sufficiently close to $-\gamma$, then under conditions (3.64)-3.65), both coefficients are positive and bounded away from 0 uniformly in $x_{3}$. This property allows us to repeat all the proofs in Section 5 with small and evident changes.

There are two subtle point is in the construction of local representatives and local almost inverses in the appendix: when the set $U_{\epsilon, j}^{2}$ intersects with the plane $x_{1}=0$, we take $x^{\epsilon, j}$ in this plane (similarly in the case of the intersection with the plane $x_{2}=0$ ), and if with the line $x_{1}=x_{2}=0$, then on this line. In the first case, we freeze all the coefficients except for the ones in the expressions $x_{1} \partial_{1}$ and $x_{1} \partial_{1}^{2}$ (similarly for the local representatives at points in the plane $x_{2}=0$ ), and in the second case, all the coefficients except for the ones in the expressions $x_{l} \partial_{l}$ and $x_{l} \partial_{l}^{2}, l=1,2$. In the first case, the model family are operators on the half-line, and in the second case, on the quadrant $\left\{x_{1}>0, x_{2}>0\right\}$. The remaining details are straightforward modifications of the corresponding steps in the proof for the family $A_{1}(2)$.

\section{Appendix A. Proof of Theorem [5.4, B)}

A.1. Reduction to construction of almost inverses. We start with the case $\rho=0$, and in the end of the proof, indicate which changes need to be made for the case of small $|\rho|$.

For each $\lambda \in \mathbf{C}$ satisfying $|\lambda| \geq \epsilon^{-1}$, introduce the family of Banach (in fact, Hilbert) spaces $H_{\lambda}^{1} \subset H:=L_{2}\left(\mathbf{R}_{+} \times \mathbf{R}\right)$, which consists of functions with the finite norm $\|\cdot\|_{H_{\lambda}^{1}}$ defined by

$$
\|u\|_{H_{\lambda}^{1}}^{2}:=\|(|x|+|\lambda|) u\|^{2}+\left\|\partial_{1} u\right\|^{2}+\left\|x_{1} \partial_{1} u\right\|^{2}+\sum_{j=0}^{2}\left(\left\|\partial_{2}^{j} u\right\|^{2}+\left\|x_{1} \partial_{2}^{j} u\right\|^{2}\right),
$$

where $\|\cdot\|$ is the norm in $H$. Notice that the embedding $H_{\lambda}^{1} \subset H$ is continuous, and $H_{\lambda}^{1}$ is independent of $\lambda$ as a topological space; but it is convenient to derive estimates for the auxiliary operators below in terms of the norm depending on the parameter.

To prove the invertibility of $-L_{\epsilon}+r_{\epsilon}+\lambda$ and estimate (4.15), it suffices to construct left and right almost inverses, $R_{\lambda, \epsilon}^{l}$ and $R_{\lambda, \epsilon}^{r}$. These are operators which are uniformly bounded

$$
\begin{aligned}
\left\|R_{\lambda, \epsilon}^{l}\right\|_{H \rightarrow H_{\lambda}^{1}} & \leq C \\
\left\|R_{\lambda, \epsilon}^{r}\right\|_{H \rightarrow H_{\lambda}^{1}} & \leq C
\end{aligned}
$$


where $C$ is independent of $\epsilon \in(0,1)$ and $\lambda \in \Sigma_{\sigma, \lambda_{0}}$, and satisfy the approximate equalities in the definition of the inverse:

$$
\begin{aligned}
& R_{\lambda, \epsilon}^{l}\left(-L_{\epsilon}+r_{\epsilon}+\lambda\right)=I+T_{\lambda, \epsilon}^{l}, \\
& \left(-L_{\epsilon}+r_{\epsilon}+\lambda\right) R_{\lambda, \epsilon}^{r}=I+T_{\lambda, \epsilon}^{r},
\end{aligned}
$$

where

$$
\begin{aligned}
\left\|T_{\lambda, \epsilon}^{r}\right\|_{H \rightarrow H} & \rightarrow 0, \quad \text { as } \epsilon \rightarrow 0, \\
\left\|T_{\lambda, \epsilon}^{l}\right\|_{H \rightarrow H_{\lambda}^{1}} & \rightarrow 0, \quad \text { as } \epsilon \rightarrow 0,
\end{aligned}
$$

uniformly w.r.t. $\lambda \in \Sigma_{\sigma, \lambda_{0}}$. Notice that (A.4) and (A.5) imply that $R_{\lambda, \epsilon}^{r}$ maps $H$ into the domain of $-L_{\epsilon}+r_{\epsilon}$. Suppose that the almost inverses are constructed, and $\epsilon$ is so small that the norms of operators $T_{\lambda, \epsilon}^{r}$ and $T_{\lambda, \epsilon}^{l}$ are less than 1/2. Then the RHS in (A.3) and (A.4) are invertible bounded operators, and hence $-L_{\epsilon}+r_{\epsilon}+\lambda$ has the bounded inverse. Moreover, the inverse $R_{\lambda, \epsilon}^{r}\left(I+T_{\lambda, \epsilon ; r}\right)^{-1}$ is a bounded operator from $H$ to $H_{\lambda}^{1}$, uniformly in $\lambda$, which implies (5.14).

Thus, it remains to construct left and right almost inverses. The standard technique consists of the construction of

(i) an appropriate partition of unity,

(ii) local representative of the operator (localization of the operator), that is, the freezing of coefficients w.r.t. to all the variables or some of them at some points,

(iii) the inverses to the local representatives, and finally,

(iv) global almost inverses by using the partition of unity and the local inverses.

Notice that $\Sigma_{\sigma, \lambda_{0}}$ depends on $\epsilon$, and $|\lambda| \rightarrow+\infty$ as $\epsilon \rightarrow 0$, uniformly in $\lambda \in \Sigma_{\sigma, \lambda_{0}}$; this property will be used systematically in the constructions below.

A.2. Partition of unity. We use the universal construction due to Hörmander (1985), which is based on the definition of a slowly varying metric; for the variant of the construction for operators with parameter, see Levendorskii (1993). Fix $\omega \in(0,1 / 2)$, and for each $\epsilon \in(0,1)$, define a function $\langle y\rangle_{\epsilon}=\left(|\epsilon|^{-2}+y^{2}\right)^{1 / 2}$ on $\mathbf{R}$, and the Riemann metric $G_{\epsilon}$ on on $R^{2}$ by

$$
G_{\epsilon ; x}(z)=\left\langle x_{1}\right\rangle_{\epsilon}^{-2 \omega}\left|z_{1}\right|^{2}+\left\langle x_{2}\right\rangle_{\epsilon}^{-2 \omega}\left|z_{2}\right|^{2} .
$$

It is straightforward to check that the derivatives of the function $\langle y\rangle_{\epsilon}^{\omega}$ w.r.t. $y$ are bounded uniformly in $y \in \mathbf{R}^{2}$ and $\epsilon \in(0,1)$, therefore (see Levendorskii (1993)) there exist $c, c_{1}, C_{1}>0$ such that if $G_{\epsilon ; x}(x-z) \leq c$, then

$$
c_{1} G_{\epsilon ; z}(w) \leq G_{\epsilon ; x}(w) \leq C_{1} G_{\epsilon ; z}(w), \quad \forall w \in \mathbf{R}^{2} .
$$


Condition A.7 means that the metric $G_{\epsilon}$ is slowly varying (uniformly w.r.t. $\epsilon \in(0,1))$, and therefore there exist positive constants $C_{2}, C_{3}, C_{4}$, points $x^{\epsilon, j} \in \mathbf{R}^{2}$, and non-negative functions $\nu_{\epsilon, j} \in C^{\infty}\left(\mathbf{R}^{2}\right), j=$ $1,2, \ldots$, such that

(i) sets $U_{\epsilon, j}=\operatorname{supp} \nu_{\epsilon, j}, j=1,2, \ldots$, cover $\mathbf{R}^{2}$;

(ii) the multiplicity of the covering $\left\{U_{\epsilon, j}\right\}_{j \geq 1}$ is bounded uniformly w.r.t. $\epsilon \in(0,1)$, and $j=1,2, \ldots$, that is, for each $j$, the number of $k$ for which $U_{\epsilon, j}$ and $U_{\epsilon, k}$ intersect is bounded by $C_{2}$;

(iii) $x^{\epsilon, j} \in U_{\epsilon, j}$;

(iv) the diameter of $U_{\epsilon, j}$ is not greater than $C_{2}\left(|\epsilon|^{-1}+\left|x^{\epsilon, j}\right|\right)^{\omega}$;

(v) for all multi-indices $s, \epsilon \in(0,1)$, and $j \geq 1$,

$$
\left|\partial^{s} \nu_{\epsilon, j}(x)\right| \leq C_{s}\left\langle x^{\epsilon, j}\right\rangle_{\epsilon}^{-|s| \omega}, \quad \forall x
$$

where $|s|=s_{1}+s_{2}$, and the constants $C_{s}$ are independent of $\epsilon \in(0,1)$, and $j \geq 1$

(vi) for all $\epsilon \in(0,1)$, and all $x \in \mathbf{R}^{2}$,

$$
\sum_{j \geq 1} \nu_{\epsilon, j}(x)=1 \text {. }
$$

For details of the construction, see Hörmander (1985) and Levendorskii (1993). In the construction, one can choose the points and functions so that

(vii) either $x^{\epsilon, j}$ is on the line $x_{1}=0$, or $x^{\epsilon, j}$ and $U_{\epsilon, j}$ are outside the strip $\left|x_{1}\right| \leq c_{2}|\epsilon|^{-\omega}$, where $c_{2}>0$ is independent of $\epsilon \in(0,1)$ and $j$.

From now on, we assume that (i)-(vii) hold, and we consider only the points in the half-plane $x_{1} \geq 0$; now the $\nu_{\epsilon, j}$ are functions defined in the same half-plane, and (A.9) holds for $x$ from this half-plane. We divide the set of the points $x^{\epsilon, j}$ in the half-plane $x_{1} \geq 0$ into two subsets: $j \in J_{0}$, if $x^{\epsilon, j}$ is on the boundary $x_{1}=0$, and $j \in J_{+}$, if $x^{\epsilon, j}$ belongs to the open half-plane $x_{1}>0$.

Construct

$$
\nu_{\epsilon, j}^{1}=\sum_{k: U_{\epsilon, k} \cap U_{\epsilon, j} \neq \emptyset} \nu_{\epsilon, k},
$$

and set $U_{\epsilon, j}^{1}=\operatorname{supp} U_{\epsilon, j}$. Similarly, starting with $\nu_{\epsilon, j}^{1}$ and $U_{\epsilon, j}^{1}$, construct $\nu_{\epsilon, j}^{2}$ and $U_{\epsilon, j}^{2}$ Then $\nu_{\epsilon, j}^{l}$ and $U_{\epsilon, j}^{l}, l=1,2$, satisfy the same conditions as $\nu_{\epsilon, j}$ and $U_{\epsilon, j}$ (with different constants) but (A.9). In addition,

$$
\begin{aligned}
& \nu_{\epsilon, j}^{1}(x)=1, \quad \forall x \in U_{\epsilon, j}, \\
& \nu_{\epsilon, j}^{2}(x)=1, \quad \forall x \in U_{\epsilon, j}^{1} .
\end{aligned}
$$


A.3. Local inverses to non-degenerate local representatives. For $j \in J_{+}$, denote by $A_{\lambda, \epsilon, j}$ the operator $-L_{\epsilon}+r_{\epsilon}+\lambda$ with the coefficients freezed at $x^{\epsilon, j}$ :

$$
A_{\lambda, \epsilon, j}=-\theta_{1} \partial_{1}-\frac{1}{2} x_{1}^{\epsilon, j} \partial_{1}^{2}+\mu_{\epsilon}\left(x^{\epsilon, j}\right) \partial_{2}-\frac{\alpha+\beta x_{1}^{\epsilon, j}}{2} \partial_{2}^{2}+r_{\epsilon}\left(x^{\epsilon, j}\right)+\lambda .
$$

The function

$$
a_{\lambda, \epsilon, j}(\xi)=-i \theta_{1} \xi_{1}+\frac{1}{2} x_{1}^{\epsilon, j} \xi_{1}^{2}+i \mu_{\epsilon}\left(x^{\epsilon, j}\right) \xi_{2}+\frac{\alpha+\beta x_{1}^{\epsilon, j}}{2} \xi_{2}^{2}+r_{\epsilon}\left(x^{\epsilon, j}\right)+\lambda
$$

is the symbol of the operator $A_{\lambda, \epsilon, j}$, that is,

$$
A_{\lambda, \epsilon, j}=a_{\lambda, \epsilon, j}(D):=\mathcal{F}^{-1} a_{\lambda, \epsilon, j}(\xi) \mathcal{F},
$$

where $\mathcal{F}$ is the Fourier transform. Equivalently, for a sufficiently regular function $u$,

$$
a_{\lambda, \epsilon, j}(D) u(x)=(2 \pi)^{-2} \int_{\mathbf{R}^{2}} e^{i\langle x, \xi\rangle} a_{\lambda, \epsilon, j}(\xi) \hat{u}(\xi) d \xi,
$$

where

$$
\hat{u}(\xi)=\int_{\mathbf{R}^{2}} e^{-i\langle x, \xi\rangle} u(x) d x
$$

is the Fourier transform of $u$.

Lemma A.1. There exist $c>0$ and $\epsilon_{0}>0$ such that for all $\epsilon \in\left(0, \epsilon_{0}\right)$, $\lambda \in \Sigma_{\sigma, \lambda_{0}}, j \in J_{+}$and $\xi \in \mathbf{R}^{2}$,

$$
\left|a_{\lambda, \epsilon, j}(\xi)\right| \geq c\left(|\lambda|+\left|x^{\epsilon, j}\right|+x_{1}^{\epsilon, j}|\xi|^{2}\right) .
$$

Proof. Due to our choice of $\sigma$,

$$
\Re \lambda>-\epsilon_{1}|\Im \lambda|, \quad \forall \lambda \in \Sigma_{\sigma, \lambda_{0}},
$$

where $\epsilon_{1} \rightarrow 0$ as $\epsilon \rightarrow 0$. Hence, if $C>0$ is fixed, then for all $\lambda \in \Sigma_{\sigma, \lambda_{0}}$, $j \in J_{+}$and $\xi \in \mathbf{R}^{2}$, satisfying

$$
|\lambda| \leq C\left(\left|x^{\epsilon, j}\right|+x_{1}^{\epsilon, j}|\xi|^{2}\right),
$$

we have

$$
\Re a_{\lambda, \epsilon, j}(\xi) \geq \frac{1}{2} x_{1}^{\lambda, j} \xi_{1}^{2}+\frac{\alpha+\beta x_{1}^{\epsilon, j}}{2} \xi_{2}^{2}+r_{\epsilon}\left(x^{\epsilon, j}\right)-C \epsilon_{1}\left(\left|x^{\epsilon, j}\right|+x_{1}^{\epsilon, j}|\xi|^{2}\right),
$$

and for sufficiently small $\epsilon>0$, (A.12) obtains. On the other hand, if

$$
|\lambda| \geq C\left(\left|x^{\epsilon, j}\right|+x_{1}^{\epsilon, j}|\xi|^{2}\right),
$$

then $\left|a_{\lambda, \epsilon, j}(\xi)-\lambda\right||\lambda|^{-1} \rightarrow 1$, as $C \rightarrow+\infty$, and hence if $C$ is large, and $\lambda \in \Sigma_{\sigma, \lambda_{0}}, j \in J_{+}$and $\xi \in \mathbf{R}^{2}$ satisfy condition (A.15), then (A.12) holds as well. 
In view of (A.12), we can define pseudo-differential operators (PDO) $R_{\lambda, \epsilon, j}, j \in J_{+}$, by

$$
R_{\lambda, \epsilon, j}=a_{\lambda, \epsilon, j}(D)^{-1}:=\mathcal{F}^{-1} a_{\lambda, \epsilon, j}(\xi)^{-1} \mathcal{F} .
$$

By using the Fourier transform, we immediately obtain that $R_{\lambda, \epsilon, j}$ is the inverse to $A_{\lambda, \epsilon, j}$ :

$$
A_{\lambda, \epsilon, j} R_{\lambda, \epsilon, j}=I, \quad R_{\lambda, \epsilon, j} A_{\lambda, \epsilon, j}=I .
$$

A.4. Local inverses to degenerate local representatives. For $j \in$ $J_{0}$, define

$$
A_{\lambda, \epsilon, j}=-\theta_{1} \partial_{1}-\frac{1}{2} x_{1} \partial_{1}^{2}-\frac{\alpha+\beta x_{1}}{2} \partial_{2}^{2}+r_{\epsilon}\left(x^{\epsilon, j}\right)+\lambda,
$$

and introduce the Hilbert space $H_{\lambda, 0}$ of functions on the half-plane $x_{1}>0$, with the finite norm $\|\cdot\|_{\lambda, \epsilon, j}$ defined by

$\|u\|_{\lambda, \epsilon, j}^{2}=\left\|\partial_{1} u\right\|^{2}+\left\|x_{1} \partial_{1}^{2} u\right\|^{2}+\sum_{s=0}^{2}\left(\left\|\partial_{2}^{s} u\right\|^{2}+\left\|x_{1} \partial_{2}^{s} u\right\|^{2}\right)+\left\|\left(\left|x_{2}^{\epsilon, j}\right|+|\lambda|\right) u\right\|^{2}$, where $\|\cdot\|$ is the norm in $H=L_{2}\left(\mathbf{R}_{+} \times \mathbf{R}\right)$.

Lemma A.2. There exist $\epsilon_{0}>0$ and $C$ such that for each $\lambda \in \Sigma_{\sigma, \lambda_{0}}$, the operator $A_{\lambda, \epsilon, j}: H_{\lambda, \epsilon, j} \rightarrow H$ is invertible, and the norm of the inverse, call it $R_{\lambda, \epsilon, j}$, is bounded by $C$.

Proof. This a very special case of general results for degenerate elliptic operators in Levendorskii (1993). We recall the scheme of the proof. First, we make the (partial) Fourier transform $\tilde{\mathcal{F}}$ w.r.t. to $x_{2}$, and obtain

$$
A_{\lambda, \epsilon, j}=\tilde{\mathcal{F}}^{-1} A_{\lambda, \epsilon, j}\left(\xi_{2}\right) \tilde{\mathcal{F}}
$$

where

$$
A_{\lambda, \epsilon, j}\left(\xi_{2}\right)=-\theta_{1} \frac{d}{d y}-\frac{1}{2} y \frac{d^{2}}{d y^{2}}+\frac{\alpha+\beta y}{2} \xi_{2}^{2}+r_{\epsilon}\left(x^{\epsilon, j}\right)+\lambda
$$

is the family of operators on $\mathbf{R}_{+}$parametrized by $\xi_{2} \in \mathbf{R}$ and $\lambda \in \Sigma_{\sigma, \lambda_{0}}$. Introduce the Hilbert space $H_{\lambda, \epsilon, j, \xi_{2}}$ of functions on $\mathbf{R}_{+}$, with the finite norm $\|\cdot\|_{\lambda, \epsilon, j, \xi_{2}}$ defined by

$$
\|u\|_{\lambda, \epsilon, j, \xi_{2}}^{2}=\left\|u^{\prime}\right\|^{2}+\left\|y u^{\prime \prime}\right\|^{2}+\left\|(1+y) \xi^{2} u\right\|^{2}+\left\|\left(\left|x_{2}^{\epsilon, j}\right|+|\lambda|\right) u\right\|^{2},
$$

where $\|\cdot\|$ is the norm in $L_{2}\left(\mathbf{R}_{+}\right)$. By using an appropriate partition on unity on $\mathbf{R}_{+}$, which depends on $\left(\lambda, \xi_{2}\right)$, one shows that if $\epsilon_{0}$ is sufficiently small then for all $\epsilon \in\left(0, \epsilon_{0}\right), \lambda \in \Sigma_{\sigma, \lambda_{0}}$, and $\xi_{2} \in \mathbf{R}$, operator

$$
A_{\lambda, \epsilon, j}\left(\xi_{2}\right): H_{\lambda, \epsilon, j, \xi_{2}} \rightarrow L_{2}\left(\mathbf{R}_{+}\right)
$$


is invertible, and the norm of the inverse, $R_{\lambda, \epsilon, j}\left(\xi_{2}\right)$, is bounded by a constant which is independent of $\lambda, \epsilon, \xi_{2}$.

It remains to define

$$
R_{\lambda, \epsilon, j}=\tilde{\mathcal{F}}^{-1} R_{\lambda, \epsilon, j}\left(\xi_{2}\right) \tilde{\mathcal{F}}
$$

A.5. Construction of almost inverses. Set

$$
\begin{aligned}
& R_{\lambda, \epsilon}^{l}=\sum_{j \geq 1} \nu_{\epsilon, j} R_{\lambda, \epsilon, j} \nu_{\epsilon, j}^{1}, \\
& R_{\lambda, \epsilon}^{r}=\sum_{j \geq 1} \nu_{\epsilon, j}^{1} R_{\lambda, \epsilon, j} \nu_{\epsilon, j} .
\end{aligned}
$$

We check that if $\epsilon_{0}>0$ is sufficiently small then the conditions (A.2), (A.4) and (A.5) hold for the family of almost right inverses $R_{\lambda, \epsilon}^{r}$; the verification of their analogs for almost left inverses $R_{\lambda, \epsilon}^{l}$ is similar.

Notice that

1) the multiplicity of the covering $\left\{U_{\epsilon, j}^{2}\right\}_{j \geq 1}$ is finite;

2) the norms are defined in terms of integration of a function and its derivatives multiplied by weight functions;

3) the derivatives of functions $\nu_{\epsilon, j}^{s}$ satisfy estimates (A.8), and

4) (A.9), (A.10) and (A.11) hold.

Therefore, it suffices to prove that

a) there exist $\epsilon_{0}>0$ and $C$ such that for all $\epsilon \in\left(0, \epsilon_{0}\right), \lambda \in \Sigma_{\sigma, \lambda_{0}}$, and $j \geq 1$

$$
\left\|\nu_{\epsilon, j}^{1} R_{\lambda, \epsilon, j}\right\|_{H \rightarrow H_{\lambda}^{1}} \leq C
$$

b) let $\epsilon_{0} \rightarrow 0$; then uniformly in $\epsilon \in\left(0, \epsilon_{0}\right), \lambda \in \Sigma_{\sigma, \lambda_{0}}$, and $j \geq 1$,

$$
\left.\| \nu_{\epsilon, j}^{2}\left(-L_{\epsilon}+r_{\epsilon}+\lambda-A_{\lambda, \epsilon, j}\right) \nu_{\epsilon, j}^{1}\right) \|_{H_{\lambda}^{1} \rightarrow H} \rightarrow 0 ;
$$

and

$$
\left\|\nu_{\epsilon, j}^{2}\left(\nu_{\lambda, j}^{1} A_{\lambda, \epsilon, j}-A_{\lambda, \epsilon, j} \nu_{\epsilon, j}^{1}\right)\right\|_{H_{\lambda}^{1} \rightarrow H} \rightarrow 0 .
$$

Indeed, (A.2) follows from (A.17) since the multiplicity of the covering $\left\{U_{\epsilon, j}^{1}\right\}_{j \geq 1}$, call it $M$, is finite: for any $u \in H$,

$$
\begin{aligned}
\left\|R_{\lambda, \epsilon, r} u\right\|_{H_{\lambda}^{1}} & \leq M \sup _{j}\left\|\nu_{\epsilon, j}^{1} R_{\lambda, \epsilon, j} \nu_{\epsilon, j} u\right\|_{H} \\
& \leq M C \sup _{j}\left\|\nu_{\epsilon, j} u\right\|_{H} \\
& =M C\|u\|_{H} .
\end{aligned}
$$


Further, by using (A.11), we can write

$$
\begin{aligned}
\left(-L_{\epsilon}+r_{\epsilon}+\lambda\right) R_{\lambda, \epsilon, r}= & \sum_{j \geq 1} \nu_{\epsilon, j}^{1} A_{\lambda, \epsilon, j} R_{\lambda, \epsilon, j} \nu_{\epsilon, j} \\
& +\sum_{j \geq 1} \nu_{\epsilon, j}^{2}\left[\nu_{\lambda, j}^{1} A_{\lambda, \epsilon, j}-A_{\lambda, \epsilon, j} \nu_{\lambda, j}^{1}\right] R_{\lambda, \epsilon, j} \nu_{\epsilon, j} \\
& \left.+\sum_{j \geq 1} \nu_{\epsilon, j}^{2}\left(-L_{\epsilon}+r_{\epsilon}+\lambda-A_{\lambda, \epsilon, j}\right) \nu_{\epsilon, j}^{1}\right) R_{\lambda, \epsilon, j} \nu_{\epsilon, j} .
\end{aligned}
$$

By construction, $A_{\lambda, \epsilon, j} R_{\lambda, \epsilon, j}=I$, therefore due to (A.9) and (A.10), the first sum on the RHS of (A.20) is identity:

$$
\sum_{j \geq 1} \nu_{\epsilon, j}^{1} A_{\lambda, \epsilon, j} R_{\lambda, \epsilon, j} \nu_{\epsilon, j}=\sum_{j \geq 1} \nu_{\epsilon, j}^{1} \nu_{\epsilon, j}=I .
$$

Since the multiplicity of the covering $\left\{U_{\epsilon, j}^{2}\right\}_{j \geq 1}$ is finite, we conclude from (A.17), A.18) and A.19), that the norms of the last two sums on the RHS of (A.20), as operators in $H$, tends to 0 as $\epsilon_{0} \rightarrow 0$, uniformly in $\epsilon \in\left(0, \epsilon_{0}\right), \lambda \in \Sigma_{\sigma, \lambda_{0}}$. This proves (A.4) and (A.5), and finishes the proof of part b) of Theorem 5.4.

A.6. Proof of estimates (A.17), (A.18) and (A.19). It suffices to use the following simple observations:

1. Due to the choice $\omega \in(0,1 / 2)$, for $j \in J_{0}$, (5.8) gives

$$
\left\|\left(r_{\epsilon}(x)-r_{\epsilon}\left(x^{\epsilon, j}\right)\right) \nu_{\epsilon, j}^{1}\right\|_{H_{\lambda}^{1} \rightarrow H} \rightarrow 0
$$

as $\epsilon \rightarrow 0$, uniformly in $j$;

2. Since function $\mu_{\epsilon}$ is an affine function of $x_{1}$ with coefficients uniformly bounded w.r.t. $x_{2}, \epsilon \in(0,1)$ and the other parameters, and the coefficients satisfy (5.11), we conclude that

$$
\left\|\mu_{\epsilon} \nu_{\epsilon, j}^{1} \partial_{2}\right\|_{H_{\lambda}^{1} \rightarrow H} \rightarrow 0
$$

as $\epsilon \rightarrow 0$, uniformly in $j$;

3. For $j \in J_{+}$, an error of freezing of any coefficient at $x^{\epsilon, j}$, on the set $U_{\epsilon, j}^{2}$ produces a relative error of order $\epsilon^{\omega}$;

4. Any derivative of any $\nu_{\epsilon, j}$ and $\nu_{\epsilon, j}^{l}$ is of order $\epsilon^{\omega}$, and hence each time we calculate such a derivative, the term, in which the derivative enters, has a small norm.

A.7. Proof of (4.17) for small $\rho$. Set $L_{\epsilon, \rho}=\exp \left(\rho\left\langle x_{2}\right\rangle\right) L_{\epsilon} \exp \left(-\rho\left\langle x_{2}\right\rangle\right)$. Direct calculations show that $L_{\epsilon, \rho} \rightarrow L_{\epsilon}$ as $\rho \rightarrow 0$, in the operator norm, uniformly in $\epsilon$, therefore for small $\rho$, the almost inverses to $\lambda-L_{\epsilon}+r_{\epsilon}$ can be used to construct the inverses to $\lambda-L_{\epsilon, \rho}+r_{\epsilon}$, and the rest of the proof of (4.17) remains the same. 


\section{REFERENCES}

[1] Chacko, G., and S. Das (2002) "Pricing interest rate derivatives: a general approach", Review of Financial Studies, 15:1, 195-241.

[2] Cox, J.C., J.E. Ingersoll, and S.A. Ross (1985) "A theory of term structure of interest rates", Econometrica, 53, 385-407.

[3] Dai, Qiang, and K.J. Singleton (2000) "Specification analysis of affine term structure models", Journal of Finance, 55:5, 1943-1978.

[4] Duffie, D., and R. Kan (1996) "A yield-factor model of interest rates", Mathematical Finance, 6, 376-406.

[5] Duffie, D., J. Pan, and K. Singleton (2000) "Transform analysis and option pricing for affine jump-diffusions", Econometrica, 68, 1343-1376.

[6] Duffie, D., D. Filipović, and W. Schachermayer (2002) "Affine processes and applications in Finance", Annals of Applied Probability

[7] Heston, S. (1993) "A closed form solution for options with stochastic volatility with applications to bond and currency options", Review of Financial Studies, $6,327-423$.

[8] Hörmaner, L. (1985). Analysis of Partial Differential Opertors. Vol. III, Springer-Verlag, Berlin Heidelberg New-York.

[9] Levendorskiï, S. (1993) Degenerate elliptic equations. Mathematics and its Applications, 258. Kluwer Academic Publishers Group, Dordrecht, xii+431 pp.

[10] Levendorskií, S. Z., and B. P. Paneyakh (1990) "Degenerate elliptic equations and boundary value problems", in Current problems in mathematics. Fundamental directions, Vol. 63 (Russian), 131-200; Translation in Encyclopaedia of Mathematical Sciences, v.63, 131-202 (1994), Springer-Verlag

[11] Sato, K. (1999). Lévy Processes and Infinitely Divisible Distributions. Cambridge: Cambridge University Press.

[12] Vasicek, O. (1977) "An equilibrium characterization of the term structure", Journ. of Financial Economics, 5, 177-188.

[13] Yosida, K. (1964). Functional Analysis. Springer-Verlag, Berlin Heidelberg New-York. 